\title{
Two-body and Three-body Decays of Charginos in One-loop Order in MSSM 1
}

\author{
FUJIMOTO Junpei ${ }^{a}$, ISHIKAWA Tadashi ${ }^{a}$, \\ JIMBO Masato ${ }^{b}, \mathrm{KON}$ Tadashi ${ }^{c}$, \\ KURIHARA Yoshimasa ${ }^{a}$, KURODA Masaaki ${ }^{d}$ \\ ${ }^{a}$ KEK, Oho, Tsukuba, Ibaraki 305-0801 Japan \\ ${ }^{b}$ Tokyo Management College, Ichikawa, Chiba 272-0001, Japan \\ ${ }^{c}$ Seikei University, Musashino, Tokyo 180-8633, Japan \\ ${ }^{d}$ Meiji Gakuin University, Totsuka, Yokohama 244-8539, Japan
}

\begin{abstract}
We present the renormalization scheme used in and the characteristic features of GRACE/SUSY-loop, the package of the program for the automatic calculation of the MSSM processes including one-loop order corrections. The twobody and three-body decay widths of charginos in one-loop order evaluated by GRACE/SUSY-loop are shown.
\end{abstract}

\section{Introduction}

The concept of supersymmetry (SUSY) [1, 2] is considered the most promising extension of the Standard Model (SM) [3] of particle physics. Among the supersymmetric theories, the minimal supersymmetric standard model (MSSM) 2] is the most elaborated and well studied framework of SUSY. The existence of many new supersymmetric particles in any SUSY model makes it very complicated and tedious to compute even a simple two-body decay width exactly. To overcome this problem, the Minami-tateya group of KEK has constructed a computational system GRACE/SUSY [4, 5] which automatically creates, for a given process, all the Feynman diagrams and compute the

\footnotetext{
${ }^{1}$ Dedicated to Dr. J. Kodaira, our friend and colleague, who passed away in Sept. 2006
} 
Feynman amplitudes and subsequently the cross section or the decay width itself at tree level in the MSSM.

With the increase of more precise experimental data, in particular, in future colliders, it becomes apparent that the inclusion of at least one-loop radiative corrections is necessary for calculations of cross sections of SUSY processes. For this purpose, the SPA project has established the convention of the SUSY parameters and loop calculations [6]. We have, therefore, extended GRACE/SUSY in order to incorporate radiative corrections in oneloop order in the system. The new system, called GRACE/SUSY-1oop, is constructed based on the same philosophy as for GRACE-1oop [7] developed by the Minami-tateya group, which is the automatic computation system for the SM processes including one-loop corrections. Some of the results computed by this system have already been presented in several workshops and symposiums [8, 9, 10].

In this paper we present the result of the thorough investigation of the two-body and three-body decays of charginos in one-loop order in the MSSM computed by the automatic computing system GRACE/SUSY-loop. We also show the cross section of the chargino pair production in $e^{+} e^{-}$annihilation and the subsequent decays by combining the production cross section and the decay rates.

The paper is organized as follows. In section two, the renormalization scheme used in GRACE/SUSY-1oop is explained. The features of the GRACE/SUSY-loop system are briefly given in section three. The numerical results are presented in section four, and several comments on the numerical results are given in section five.

\section{Renormalization scheme}

In this section we explain briefly the renormalization scheme adopted in GRACE/SUSY-1oop. Our approach is a straightforward extension of the onshell renormalization in the SM used in GRACE [7].

The Lagrangian of the MSSM has been given in [11, 12, 13]. In terms of superfields, it is given as ( see [11] for detail)

$$
\begin{aligned}
\mathcal{L}= & \int d^{2} \theta \frac{1}{4}\left[2 \operatorname{Tr}(\mathbf{W} \mathbf{W})+W W+2 \operatorname{Tr}\left(\mathbf{W}_{\mathbf{s}} \mathbf{W}_{\mathbf{s}}\right)\right]+\text { h.c. } \\
& +\int d^{2} \theta d^{2} \bar{\theta} \boldsymbol{\Phi}_{\ell}^{\dagger} \exp \left[2\left(g \frac{\tau^{a}}{2} V^{a}+g^{\prime} \frac{Y_{\ell}}{2} V\right)\right] \mathbf{\Phi}_{\ell}
\end{aligned}
$$




$$
\begin{aligned}
& +\int d^{2} \theta d^{2} \bar{\theta} \Phi_{e}^{\dagger} \exp \left(g^{\prime} Y_{e} V\right) \Phi_{e} \\
& +\int d^{2} \theta d^{2} \bar{\theta} \boldsymbol{\Phi}_{\mathbf{q}}^{\dagger} \exp \left[2\left(g \frac{\tau^{a}}{2} V^{a}+g^{\prime} \frac{Y_{q}}{2} V+g_{s} \frac{\lambda^{\alpha}}{2} V_{s}^{\alpha}\right)\right] \boldsymbol{\Phi}_{\mathbf{q}} \\
& +\int d^{2} \theta d^{2} \bar{\theta} \Phi_{u}^{\dagger} \exp \left(g^{\prime} Y_{u} V-g_{s} \lambda^{\alpha *} V_{s}^{\alpha}\right) \Phi_{u} \\
& +\int d^{2} \theta d^{2} \bar{\theta} \Phi_{d}^{\dagger} \exp \left(g^{\prime} Y_{d} V-g_{s} \lambda^{\alpha *} V_{s}^{\alpha}\right) \Phi_{d} \\
& +\int d^{2} \theta d^{2} \bar{\theta} \boldsymbol{\Phi}_{\mathbf{H} \mathbf{1}}^{\dagger} \exp \left[2\left(g T^{a} V^{a}+g^{\prime} \frac{Y_{H 1}}{2} V\right)\right] \boldsymbol{\Phi}_{\mathbf{H 1}} \\
& +\int d^{2} \theta d^{2} \bar{\theta} \mathbf{\Phi}_{\mathbf{H 2}}{ }^{\dagger} \exp \left[2\left(g T^{a} V^{a}+g^{\prime} \frac{Y_{H 2}}{2} V\right)\right] \mathbf{\Phi}_{\mathbf{H 2}} \\
& +\frac{\sqrt{2} m_{e}}{v_{1}} \int d^{2} \theta \boldsymbol{\Phi}_{\mathbf{H}_{\mathbf{1}}} \boldsymbol{\Phi}_{\ell} \Phi_{e}+\text { h.c. } \\
& -\frac{\sqrt{2} m_{u}}{v_{2}} \int d^{2} \theta \boldsymbol{\Phi}_{\mathbf{H}_{\mathbf{2}}} \boldsymbol{\Phi}_{\mathbf{q}} \Phi_{u}+\text { h.c. } \\
& +\frac{\sqrt{2} m_{d}}{v_{1}} \int d^{2} \theta \boldsymbol{\Phi}_{\mathbf{H}_{\mathbf{1}}} \boldsymbol{\Phi}_{\mathbf{q}} \Phi_{d}+\text { h.c. } \\
& -\mu \int d^{2} \theta \boldsymbol{\Phi}_{\mathbf{H 1}} \boldsymbol{\Phi}_{\mathbf{H} \mathbf{2}}+\text { h.c. } \\
& +\mathcal{L}_{\text {soft }} \\
& +\mathcal{L}_{g f}+\mathcal{L}_{\text {ghost }},
\end{aligned}
$$

where $\mathbf{W}, W, \mathbf{W}_{\mathbf{s}}$ are superfield strengths corresponding to the $\mathrm{SU}(2)_{L}, \mathrm{U}(1)$ and $\mathrm{SU}(3)_{c}$ gauge-superfields, $\mathbf{V}, V, \mathbf{V}_{\mathbf{s}}$, respectively. The component fields belonging to each superfield are given as follows

$$
\begin{array}{rlr}
\mathbf{V} & =\left(\vec{W}_{\mu}, \vec{\lambda}\right), & S U(2)_{L} \text { gauge boson } \\
V & =\left(B_{\mu}, \lambda\right), & U(1) \text { gauge boson } \\
\mathbf{V}_{\mathbf{s}} & =\left(g_{\mu}^{\alpha}, \tilde{g}_{\mu}^{\alpha}\right), & S U(3)_{c} \text { gauge boson } \\
\mathbf{\Phi}_{\mathbf{H}_{\mathbf{1}}} & =\left(\left(\begin{array}{c}
\mathbf{H}_{1} \\
\tilde{\mathbf{H}}_{1}
\end{array}\right)\right)=\left(\left(\begin{array}{c}
H_{1}^{0} \\
H_{1}^{-}
\end{array}\right),\right. & \left.\left(\begin{array}{c}
\tilde{H}_{1}^{0} \\
\tilde{H}_{1}^{-}
\end{array}\right)\right), \\
\mathbf{\Phi}_{\mathbf{H}_{\mathbf{2}}} & =\left(\left(\begin{array}{c}
\mathbf{H}_{2} \\
\tilde{\mathbf{H}}_{2}
\end{array}\right)\right)=\left(\left(\begin{array}{c}
H_{2}^{+} \\
H_{2}^{0}
\end{array}\right), \quad\left(\begin{array}{c}
\tilde{H}_{2}^{+} \\
\tilde{H}_{2}^{0}
\end{array}\right)\right), \\
\Phi_{\ell} & =\left(\left(\begin{array}{c}
\nu_{L} \\
e_{L}
\end{array}\right), \quad\left(\begin{array}{c}
\tilde{\nu}_{L} \\
\tilde{e}_{L}
\end{array}\right)\right), \\
\Phi_{e} & =\left(e_{R}, \tilde{e}_{R}^{*}\right),
\end{array}
$$




$$
\begin{aligned}
\Phi_{q} & =\left(\left(\begin{array}{c}
u_{L} \\
d_{L}
\end{array}\right), \quad\left(\begin{array}{c}
\tilde{u}_{L} \\
\tilde{d}_{L}
\end{array}\right)\right), \\
\Phi_{u} & =\left(u_{R}, \tilde{u}_{R}^{*}\right), \\
\Phi_{d} & =\left(d_{R}, \tilde{d}_{R}^{*}\right),
\end{aligned}
$$

The $\mathrm{SU}(2)_{L}$ doublet gauge-bosons and gauginos are denoted by $\vec{W}_{\mu}^{a}, \overrightarrow{\lambda^{a}}$, while the singlet gauge-boson and gaugino are denoted by $B_{\mu}$ and $\lambda$. The Higgs and higgsino doublets are denoted by $\mathbf{H}_{i}$ and $\tilde{\mathbf{H}}_{i}$ with $i=1,2$, respectively. The soft SUSY breaking terms are expressed as

$$
\begin{aligned}
\mathcal{L}_{\text {soft }}= & -\frac{1}{2} M_{1} \lambda \lambda-\frac{1}{2} M_{2} \lambda^{a} \lambda^{a}-\frac{1}{2} M_{3} \tilde{g}^{\alpha} \tilde{g}^{\alpha}+\text { h.c. } \\
& -\tilde{m}_{1}^{2} \mathbf{H}_{1}^{*} \mathbf{H}_{1}-\tilde{m}_{2}^{2} \mathbf{H}_{2}^{*} \mathbf{H}_{2}-\left(m_{12}^{2} \mathbf{H}_{1} \mathbf{H}_{2}+\text { h.c. }\right)-\sum_{\tilde{f}_{m}} \tilde{m}_{\tilde{f}_{m}}^{2} \tilde{f}_{m}^{*} \tilde{f}_{m} \\
& -\frac{\sqrt{2} m_{u}}{v_{2}} A_{u} \mathbf{H}_{\mathbf{2}} \mathbf{A}\left(q_{L}\right) A\left(u_{R}\right)+\frac{\sqrt{2} m_{d}}{v_{1}} A_{d} \mathbf{H}_{\mathbf{1}} \mathbf{A}\left(q_{L}\right) A\left(d_{R}\right)+\text { h.c. } \\
& +\frac{\sqrt{2} m_{e}}{v_{1}} A_{e} \mathbf{H}_{\mathbf{1}} \mathbf{A}\left(\ell_{L}\right) A\left(e_{R}\right)+\text { h.c. }
\end{aligned}
$$

where the sum in the sfermion mass terms runs for $\tilde{f}_{m}=\tilde{f}_{L}$ and $\tilde{f}_{R}$. The sign convention of our $A_{f}$ is opposite to the convention used by others.

Sfermion mass eigenstates are denoted by $\tilde{f}_{i}$ with $i=1,2$ which are the mixture of the left-handed $\left(\tilde{f}_{L}\right)$ and the right-handed sfermions $\left(\tilde{f}_{R}\right)$. The sfermion mass matrix is diagonalized as,

$$
\begin{aligned}
\left(\begin{array}{cc}
\cos \theta_{f} & \sin \theta_{f} \\
-\sin \theta_{f} & \cos \theta_{f}
\end{array}\right) & \left(\begin{array}{cc}
m_{\tilde{f}_{L}}^{2} & m_{\tilde{f}_{L R}}^{2} \\
m_{\tilde{f}_{L R}}^{2 *} & m_{\tilde{f}_{R}}^{2}
\end{array}\right)\left(\begin{array}{cc}
\cos \theta_{f} & -\sin \theta_{f} \\
\sin \theta_{f} & \cos \theta_{f}
\end{array}\right) \\
& =\left(\begin{array}{cc}
m_{\tilde{f}_{1}}^{2} & 0 \\
0 & m_{\tilde{f}_{2}}^{2}
\end{array}\right),
\end{aligned}
$$

where

$$
\begin{aligned}
m_{\tilde{f}_{L}}^{2} & =\tilde{m}_{\tilde{f}_{L}}^{2}+m_{f}^{2}+M_{Z}^{2} \cos 2 \beta\left(T_{3 f}-Q_{f} s_{W}^{2}\right), \\
m_{\tilde{f}_{R}}^{2} & =\tilde{m}_{\tilde{f}_{R}}^{2}+m_{f}^{2}+M_{Z}^{2} \cos 2 \beta Q_{f} s_{W}^{2} \\
m_{\tilde{f}_{L R}}^{2} & = \begin{cases}-m_{u}\left(\mu \cot \beta+A_{u}\right), & f=u \\
-m_{f}\left(\mu \tan \beta+A_{f}\right), & f=d, e\end{cases}
\end{aligned}
$$


Their masses and the mixing angles satisfy the relations originating from the $\mathrm{SU}(2)_{L}$ conditions on their left-handed soft SUSY-breaking mass terms, $\tilde{m}_{\tilde{u}_{L}}^{2}=\tilde{m}_{\tilde{d}_{L}}^{2}, \tilde{m}_{\tilde{e}_{L}}^{2}=\tilde{m}_{\tilde{\nu}_{e}}^{2}$ etc. For the third generation of sfermions, for example,

$$
\begin{aligned}
\cos ^{2} \theta_{t} m_{\tilde{t}_{1}}^{2}+\sin ^{2} \theta_{t} m_{\tilde{t}_{2}}^{2}-m_{t}^{2} & =\cos ^{2} \theta_{b} m_{\tilde{b}_{1}}^{2}+\sin ^{2} \theta_{b} m_{\tilde{b}_{2}}^{2}-m_{b}^{2}+M_{W}^{2} \cos 2 \beta, \\
m_{\tilde{\nu} \tau}^{2} & =\cos ^{2} \theta_{\tau} m_{\tilde{\tau}_{1}}^{2}+\sin ^{2} \theta_{\tau} m_{\tilde{\tau}_{2}}^{2}-m_{\tau}^{2}+M_{W}^{2} \cos 2 \beta .
\end{aligned}
$$

Regarding now the Lagrangian (2.1) and (2.3) as a bare Lagrangian and renormailzing all the quantities, we separate the renormalized Lagrangian and its counterterms. The renormalization constants are introduced as follows.

[Standard Model sector]

$$
\begin{aligned}
\text { gauge bosons } \quad \vec{W}_{\mu 0} & =Z_{W}^{1 / 2} \vec{W}_{\mu}, \\
B_{\mu 0} & =Z_{B}^{1 / 2} B_{\mu}, \\
g_{\mu 0} & =Z_{\text {gluon }}^{1 / 2} g_{\mu} \\
g_{0} & =Z_{g} Z_{W}^{-3 / 2} g, \\
g_{0}^{\prime} & =Z_{g^{\prime}} Z_{B}^{-3 / 2} g^{\prime}, \\
g_{s 0} & =Z_{g_{s}} Z_{\text {gluon }}^{-3 / 2} g_{s}, \\
\Psi_{f L_{0}} & =Z_{f}^{L}{ }^{1 / 2} \Psi_{f L}, \quad f=u, d, \cdots, \nu_{e}, e, \cdots, \\
\Psi_{f R_{0}} & =Z_{f}^{R}{ }^{1 / 2} \Psi_{f R}, \quad f=u, d, \cdots, e, \cdots, \\
m_{f_{0}} & =m_{f}+\delta m_{f}, \quad f=u, d, \cdots, e, \cdots .
\end{aligned}
$$

[SUSY sector]

$$
\text { Higgs bosons } \begin{array}{rlr}
\mathbf{H}_{i 0} & =Z_{H_{i}}^{1 / 2} \mathbf{H}_{i}, & i=1,2, \\
v_{i 0} & =Z_{H_{i}}^{1 / 2}\left(v_{i}-\delta v_{i}\right), & i=1,2, \\
m_{i 0}^{2} & =Z_{H_{i}}^{-1}\left(m_{i}^{2}+\delta m_{i}^{2}\right), & i=1,2, \\
\left(m_{12}^{2}\right)_{0} & =Z_{H_{1}}^{-1 / 2} Z_{H_{2}}^{-1 / 2}\left(m_{12}^{2}+\delta m_{12}^{2}\right), \\
\text { sfermions } & \left(\begin{array}{c}
\tilde{f}_{1} \\
\tilde{f}_{2}
\end{array}\right)_{0}=\left(\begin{array}{cc}
Z_{\tilde{f}_{1} \tilde{f}_{1}}^{1 / 2} & Z_{\tilde{f}_{1} \tilde{f}_{2}}^{1 / 2} \\
Z_{\tilde{f}_{2} \tilde{f}_{1}}^{1 / 2} & Z_{\tilde{f}_{2} \tilde{f}_{2}}^{1 / 2}
\end{array}\right)\left(\begin{array}{c}
\tilde{f}_{1} \\
\tilde{f}_{2}
\end{array}\right),
\end{array}
$$




$$
\begin{array}{rlrl}
\left(\tilde{\nu}_{i}\right)_{0} & =Z_{\tilde{\nu}_{i}}^{1 / 2} \tilde{\nu}_{i}, & & f=u, d, \cdots, e, \cdots, \\
\left(m_{\tilde{f}_{i}}^{2}\right)_{0} & =m_{\tilde{f}_{i}}^{2}+\delta m_{\tilde{f}_{i}}^{2}, & & i=e, \mu, \tau, \\
& & i=u, d, \cdots, e, \cdots, \\
\left(m_{\tilde{\nu}_{i}}^{2}\right)_{0} & =m_{\tilde{\nu}_{i}}^{2}+\delta m_{\tilde{\nu}_{i}}^{2}, & & i=e, \mu, \tau, \\
\left(\theta_{f}\right)_{0} & =\theta_{f}+\delta \theta_{f}, & & f=u, d, \cdots, e, \cdots, \\
\vec{\lambda}_{0} & =Z_{\lambda^{w}}^{1 / 2} \vec{\lambda}, & \\
\lambda_{0} & =Z_{\lambda}^{1 / 2} \lambda, & \\
\tilde{\mathbf{H}}_{i 0} & =Z_{\tilde{H}_{i}}^{1 / 2} \tilde{\mathbf{H}}_{i}, & & \\
\tilde{g}_{0}^{\alpha} & =Z_{\tilde{g}}^{1 / 2} \tilde{g}^{\alpha}, & \\
\mu_{0} & =\mu+\delta \mu, 2, \\
M_{10} & =M_{1}+\delta M_{1}, & \\
M_{20} & =M_{2}+\delta M_{2}, & \\
M_{30} & =M_{3}+\delta M_{3}, &
\end{array}
$$

where in the Higgs boson part,

$$
m_{i}^{2}=\tilde{m}_{i}^{2}+|\mu|^{2}, \quad i=1,2 .
$$

Summing up, we have $\left(3+7 N_{G}\right)$ wavefunction renormalization constants, $\left(3+3 N_{G}\right)$ mass counterterms in the non-SUSY sector, and $\left(7+13 N_{G}\right)$ wavefunction renormalization constants and $\left(9+10 N_{G}\right)$ mass, vacuum-expectationvalue and mixing-angle counterterms in the SUSY sector.

Several comments are in order. The relation (2.4) $\sim(2.6)$ are originally satisfied by bare quantities, but they are also valid among the renormalized quantities, although the renormalized masses are not necessarily equal to the pole masses. In a similar way, (2.9) can be understood as the relation among the renormalized quantities. Note that at this state all the renormalized parameters in the Lagrangian are simply parameters of the model, and only upon imposing the renormalization conditions to be specified in this section, they are related to the physical quantities.

In the Higgs and higgsino sectors, the wavefunction renormalization constants are introduced to each unmixed bare doublet state. The mixing angles in the Higgs, chargino and neutralino sectors are defined as the angles which diagonalize the renormalized mass matrices. Therefore, there appear no bare 
mixing angles or counterterms for the mixing angles of charginos and neutralinos in our scheme. See [14].

In place of $\delta m_{1}^{2}, \delta m_{2}^{2}$ and $\delta m_{12}^{2}$, we use the mass counterterm of the CP odd Higgs particle, $\delta M_{A}^{2}$, and two counterterms of the tadpole interactions, $\delta \mathcal{T}_{1}$ and $\delta \mathcal{T}_{2}$, which are given by the linear combination of $\delta m_{1}^{2}, \delta m_{2}^{2}$ and $\delta m_{12}^{2}$;

$$
\begin{aligned}
\delta M_{A}^{2}= & s_{\beta}^{2} \delta m_{1}^{2}+c_{\beta}^{2} \delta m_{2}^{2}-2 c_{\beta} s_{\beta} \delta m_{12}^{2} \\
& -\frac{M_{Z}^{2}}{2}\left(c_{\beta}^{2}-s_{\beta}^{2}\right)^{2}\left[\delta Z_{Z}+\delta Z_{H_{1}}+\delta Z_{H_{2}}-\frac{2 c_{\beta}^{2}}{c_{\beta}^{2}-s_{\beta}^{2}} \frac{\delta v_{1}}{v_{1}}+\frac{2 s_{\beta}^{2}}{c_{\beta}^{2}-s_{\beta}^{2}} \frac{\delta v_{2}}{v_{2}}\right], \\
\delta \mathcal{T}_{1}= & v_{1} \delta m_{1}^{2}-m_{1}^{2} \delta v_{1}+v_{2} \delta m_{12}^{2}-m_{12}^{2} \delta v_{2} \\
& +\frac{1}{8}\left(\delta g^{2}+\delta g^{\prime 2}\right)\left(v_{1}^{2}-v_{2}^{2}\right) v_{1}+\frac{1}{8}\left(g^{2}+g^{\prime 2}\right)\left\{\left(v_{2}^{2}-3 v_{1}^{2}\right) \delta v_{1}+2 v_{1} v_{2} \delta v_{2}\right\} \\
& +\frac{1}{8}\left(g^{2}+g^{\prime 2}\right)\left\{\left(2 v_{1}^{3}-v_{1} v_{2}^{2}\right) \delta Z_{H_{1}}-v_{1} v_{2}^{2} \delta Z_{H_{2}}\right\}, \\
\delta \mathcal{T}_{2}= & (1 \leftrightarrow 2) \text { in } \delta \mathcal{T}_{1} .
\end{aligned}
$$

We introduce the gauge-fixing Lagrangian in terms of the renormalized fields as we have done in GRACE-1oop for SM [7]. No renormalization constants are introduced for gauge fixing constants and no counterterm Lagrangian corresponding to the gauge-fixing Lagrangian appears. The renormalization of the ghost fields is not necessary in one-loop order.

We use the on-shell renormalization scheme. The renormalization conditions employed in GRACE/SUSY-1oop are the following set of conditions. Using these conditions, we can express all the renormalization constants and counterterms in terms of the linear combination of the two-point functions evaluated at some specific renormalization points.

gauge sector

We use the on-mass-shell condition for $W, Z$ and photon. In addition, we require that the residue of the photon propagator at the pole position is one, and that the $A_{\mu}-Z_{\mu}$ transition vanishes for the on-shell photon.

$\underline{\mathrm{SM} \text { fermions }}$

We use the same renormalization conditions adopted in GRACE-loop. Namely, we require the on-mass-shell condition and the residue condition that the residue of the fermion propagator at the pole is one.

Higgs sector

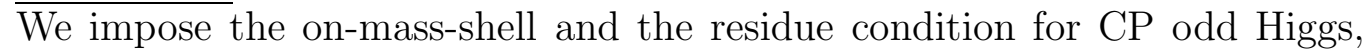


$A^{0}$, and the decoupling of $Z_{\mu}$ and $A^{0}$ on the mass-shell of $A^{0}$. For the CP even Higgs, we impose the on-mass-shell condition for the heavier Higgs, $H^{0}$. The above three conditions together with one of the conditions imposed in the gauge sector determine four renormalization constants $\delta H_{1}, \delta H_{2}, \frac{\delta v_{1}}{v_{1}}$ and $\frac{\delta v_{2}}{v_{2}}$.

Note that we have not adopted the often erroneously used renormalization condition, $\frac{\delta v_{1}}{v_{1}}=\frac{\delta v_{2}}{v_{2}}$, since this condition violates the gauge invariance [15]. tadpole terms

Identical to GRACE-loop, we require that the tadpole terms in the renormalized Lagrangian vanish by itself and the tadpole counterterms cancel the one-loop tadpole contributions.

chargino sector

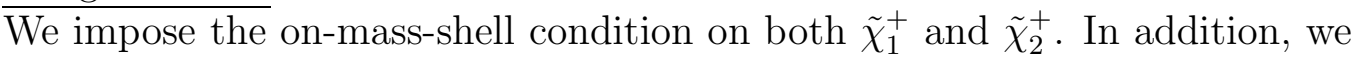
impose the residue condition on $\tilde{\chi}_{1}^{+}$.

$\underline{\text { neutralino sector }}$

We impose the on-mass-shell and the residue condition on the lightest neutralino, $\tilde{\chi}_{1}^{0}$.

sfermion sector

We impose the on-mass-shell condition and the residue condition on all the seven sfermions in each generation. In addition, we impose that there is no induced mixing between physical $\tilde{f}_{1}$ and $\tilde{f}_{2}$. The counterterm for the slepton mixing angle is determined by the $\mathrm{SU}(2)_{L}$ relation (2.6) upon introducing the renormalization constants (2.7) and (2.8). In the squark sector, there are two counterterms $\delta \theta_{u}$ and $\delta \theta_{d}$ for each generation. We fix $\delta \theta_{u}$ [16] by

$$
\delta \theta_{u}=\frac{1}{2} \frac{\sum_{\tilde{u}_{1} \tilde{u}_{2}}\left(m_{\tilde{u}_{1}}^{2}\right)+\sum_{\tilde{u}_{1} \tilde{u}_{2}}\left(m_{\tilde{u}_{2}}^{2}\right)}{m_{\tilde{u}_{2}}^{2}-m_{\tilde{u}_{1}}^{2}},
$$

while $\delta \theta_{d}$ is fixed by the $\mathrm{SU}(2)_{L}$ relation (2.6) upon introducing the renormalization constants (2.7) and (2.8).

charge renormalization

The charge (electromagnetic coupling constant) is defined as in the standard model [17.

QCD sector

We impose the on-mass-shell condition and the residue condition for gluons and gluinos. The counterterm $\delta Z_{g_{s}}$ is determined by the minimal subtraction with dimensional reduction $(\overline{\mathrm{DR}})$. 
The explicit expression of the renormalization constants in terms of twopoint functions is given in Appendix A.

A couple of comments are worthwhile at this stage. In our renormalization scheme, the pole mass of $h^{0}, H^{ \pm}, \tilde{\chi}_{2}^{0}, \tilde{\chi}_{3}^{0}$ and $\tilde{\chi}_{4}^{0}$ is different from its Born value. Therefore, the mixing angles of the Higgs sector and the neutralino sector are not directly related to the pole masses. They are considered as effective parameters. This means, in particular, that a quantity " $\tan \beta$ " which includes one-loop corrections is not defined and not used in GRACE/SUSY-loop. The relation between the parameter $\tan \beta$ used in GRACE/SUSY-loop and the experimentally observed " $\tan \beta$ " which includes higher order corrections depends on how " $\tan \beta$ " is actually defined.

In addition to the wavefunction renormalization constants introduced in (2.7) and (2.8), we need to introduce the ultraviolet finite external wavefunction renormalization constant $\delta Z^{\text {ext }}$ for each particle for which the residue condition is not imposed on its propagator, namely for $W^{ \pm}, Z^{0}, H^{0}, h^{0}, H^{ \pm}$, $\tilde{\chi}_{2}^{ \pm}, \tilde{\chi}_{2}^{0}, \tilde{\chi}_{3}^{0}, \tilde{\chi}_{4}^{0}$. The expression of $\delta Z^{\text {ext }}$ in terms of two-point functions is given in Appendix B.

Since the coupling constant of the soft SUSY-breaking Yukawa interaction among Higgs and sfermions, $A_{f}$, appears always in combination with $m_{f}$, we use in GRACE/SUSY-1oop $m_{f} A_{f}$ and its counterterm $\delta\left(m_{f} A_{f}\right)$ as independent variables, which helps avoid partly the numerical instability of the counterterm at large $\tan \beta$. Explicitly

$$
\begin{aligned}
\delta\left(m_{f} A_{f}\right)= & \frac{1}{2}\left(\delta m_{\tilde{f}_{2}}^{2}-\delta m_{\tilde{f}_{1}}^{2}\right) \sin 2 \theta_{f}+\delta \theta_{f}\left(m_{\tilde{f}_{2}}^{2}-m_{\tilde{f}_{1}}^{2}\right) \cos 2 \theta_{f} \\
& - \begin{cases}\delta\left(m_{t} \mu \cot \beta\right) & f=u, c, t \\
\delta\left(m_{f} \mu \tan \beta\right) & t=d, s, b, e, \mu, \tau\end{cases}
\end{aligned}
$$

since according to (2.4) $A_{f}$ is related to the sfermion masses $m_{\tilde{f}_{1}}^{2}$ and $m_{\tilde{f}_{2}}^{2}$ as

$$
m_{f} A_{f}=\cos \theta_{f} \sin \theta_{f}\left(m_{\tilde{f}_{2}}^{2}-m_{\tilde{f}_{1}}^{2}\right)-\left\{\begin{array}{ll}
m_{f} \mu \cot \beta & f=u, c, t \\
m_{f} \mu \tan \beta & f=d, s, b, e, \mu, \tau
\end{array} .\right.
$$

The system can easily accommodate different renormalization schemes by re-expressing the renormalization constants and the mass counterterms in terms of different linear combinations of the two-point functions.

A brief comparison of our scheme with other earlier studies is worthwhile to clarify the difference and also the possible scheme dependence in different 
schemes. There are plenty of papers on the renormalization schemes of the MSSM, and it is beyond our scope to compare all of them. The earlier works on the MSSM renormalization (see for example, [18, 14, 19]) are naturally concerned with radiative corrections in the Higgs sector. Later, the study of the renormalization of the other sector, gaugino and higgsino sector and sfermion sector followed ( see for example, [20, 21, 22, 23]). In [18, 19, 14, 22 , 24 the on-shell renormalization is used, while the so-called $\overline{D R}$ is used in [21. Since the number of the particles exceeds the number of the parameters appearing in MSSM, one cannot impose the on-shell conditions on all the particles. Therefore, there can be many variants even among the on-shell scheme, depending on which particles are put on-mass-shell.

The renormalization scheme we adopt in GRACE/SUSY-1oop is close to the scheme given by [14, but there are a couple of differences. In [14, different from our scheme, the gauge-fixing Lagrangian is introduced in terms of bare fields. Therefore, in their scheme, they need extra renormalization conditions to fix the renormalization constants for gauge-parameters. Another difference of our scheme from the others lies in the renormalization condition imposed on $\delta v_{i}$. The condition used in [19, 14, 22, $\delta v_{1} / v_{1}=\delta v_{2} / v_{2}$ is not preferable on the ground of potentially violating the Ward identity. We use the on-massshell condition for $H^{0}$ in place of the condition on the vacuum expectation value, which leads to our expression of $\delta \tan \beta$ which is also different from others.

\section{Features of GRACE/SUSY-loop}

We present in this section several features of the current version of the system GRACE/SUSY-loop. Some of them have been given in [8].

In order to check and detect possible errors in the system we have used the technique of the non-linear gauge (NLG), by adding the SUSY interactions to the gauge fixing functions of the SM [17]. Explicitly, we used the following gauge fixing functions 2

$$
\mathcal{L}_{\text {gf }}=-\frac{1}{\xi_{W}}\left|F_{W^{+}}\right|^{2}-\frac{1}{2 \xi_{Z}}\left(F_{Z}\right)^{2}-\frac{1}{2 \xi_{\gamma}}\left(F_{\gamma}\right)^{2}
$$

\footnotetext{
${ }^{2}$ In principle, we can also add non-linear sfermion interactions. We have not attempted this extension, since due to the mixing the resultant Lagrangian becomes too lengthy and cumbersome.
} 


$$
\begin{aligned}
F_{W^{ \pm}}= & \left(\partial_{\mu} \pm i e \tilde{\alpha} A_{\mu} \pm i g c_{W} \tilde{\beta} Z_{\mu}\right) W^{ \pm \mu} \\
& \pm i \xi_{W} \frac{g}{2}\left(v+\tilde{\delta}_{H} H^{0}+\tilde{\delta}_{h} h^{0} \pm i \tilde{\kappa} G^{0}\right) G^{ \pm}, \\
F_{Z}= & \partial_{\mu} Z^{\mu}+\xi_{Z} \frac{g_{Z}}{2}\left(v+\tilde{\epsilon}_{H} H^{0}+\tilde{\epsilon}_{h} h^{0}\right) G^{0}, \\
F_{\gamma}= & \partial_{\mu} A^{\mu},
\end{aligned}
$$

where

$$
v=\sqrt{v_{1}^{2}+v_{2}^{2}} .
$$

The gauge fixing functions contain now seven free parameters,

$$
\tilde{\alpha}, \quad, \tilde{\beta}, \quad \tilde{\delta}_{H}, \quad \tilde{\delta}_{h}, \quad \tilde{\kappa}, \quad \tilde{\epsilon}_{H}, \quad \tilde{\epsilon}_{h} .
$$

Since physical results are independent on the NLG parameters, they must vanish in the sum of the Feynman amplitudes for physical processes. The test using the NLG parameters provides us with a more powerful tool for the check of the system than the test using the linear gauge parameters, because each NLG parameter is concerned with many kinds of amplitudes which are not in the same gauge-independent sub-set in the linear gauge [10].

In the actual computation of decay widths and production cross sections in GRACE/SUSY-loop, we use the 't Hooft-Feynman gauge with $\xi_{V}=1$. For the consistency check of the computation, we use the vanishing of the ultraviolet divergences and the infrared singularities in the sum of the loop and the soft photon/gluon radiation diagrams, as well as the stability in the sum of the soft photon/gluon radiation and the hard photon/gluon radiation diagrams against the change of the photon/gluon energy cut-off.

The input parameters of GRACE/SUSY-loop are

$$
\begin{aligned}
& e, g_{s}, M_{W}, M_{Z}, M_{A^{0}}, \tan \beta, \mu, M_{1}, M_{2}, M_{3}, \\
& m_{u}, m_{d}, m_{e}, \cdots, \\
& m_{\tilde{u}_{1}}, m_{\tilde{u}_{2}}, m_{\tilde{d}_{1}}, m_{\tilde{d}_{2}}, m_{\tilde{e}_{1}}, m_{\tilde{e}_{2}}, m_{\tilde{\nu}_{e}}, \cdots, \\
& \theta_{u}, \theta_{d}, \theta_{e}, \cdots,
\end{aligned}
$$

Using (2.6), we fix the remaining two masses of the sfermions in each generation. Note that the width of the unstable particles is neglected in the computation of the amplitudes.

The coupling constants $A_{f}$ of the soft SUSY-breaking Yukawa interaction among Higgs and sfermions are not our independent input parame- 


\begin{tabular}{|c|c|c|c|c|c|c|c|c|c|}
\hline $\tan \beta$ & $\mu$ & $M_{1}$ & $M_{2}$ & $M_{3}$ & \multicolumn{2}{|c|}{$M_{A^{0}}$} \\
\hline 10.00 & 399.31 & 100.12 & 197.52 & 610 & \multicolumn{2}{|c|}{424.9} & \\
\hline \hline$m_{\tilde{u}_{1}}$ & $m_{\tilde{u}_{2}}$ & $m_{\tilde{d}_{1}}$ & $m_{\tilde{d}_{2}}$ & $m_{\tilde{e}_{1}}$ & $m_{\tilde{e}_{2}}$ & $m_{\tilde{\nu}_{e}}$ & $\cos \theta_{u}$ & $\cos \theta_{d}$ & $\cos \theta_{e}$ \\
\hline 545.67 & 563.44 & 545.50 & 569.03 & 125.50 & 190.14 & 172.70 & $2.4 \times 10^{-3}$ & 0.011 & $1.1 \times 10^{-4}$ \\
\hline \hline$m_{\tilde{c}_{1}}$ & $m_{\tilde{c}_{2}}$ & $m_{\tilde{s}_{1}}$ & $m_{\tilde{s}_{2}}$ & $m_{\tilde{\mu}_{1}}$ & $m_{\tilde{\mu}_{2}}$ & $m_{\tilde{\nu}_{\mu}}$ & $\cos \theta_{c}$ & $\cos \theta_{s}$ & $\cos \theta_{\mu}$ \\
\hline 545.66 & 563.45 & 545.52 & 568.97 & 125.43 & 190.16 & 172.69 & 0.063 & 0.018 & 0.023 \\
\hline \hline$m_{\tilde{t}_{1}}$ & $m_{\tilde{t}_{2}}$ & $m_{\tilde{b}_{1}}$ & $m_{\tilde{b}_{2}}$ & $m_{\tilde{\tau}_{1}}$ & $m_{\tilde{\tau}_{2}}$ & $m_{\tilde{\nu}_{\tau}}$ & $\cos \theta_{t}$ & $\cos \theta_{b}$ & $\cos \theta_{\tau}$ \\
\hline 368.53 & 583.79 & 450.12 & 544.38 & 107.71 & 195.08 & 170.63 & 0.722 & 0.967 & 0.314 \\
\hline
\end{tabular}

Table 1: The value of the MSSM input parameters for set (A)

ters, since they are expressed in terms of sfermion masses and the mixing angles as (2.15). We don't use the GUT relations $M_{1}=\frac{5}{3} \tan ^{2} \theta_{W} M_{2}$, $M_{3}=\frac{g_{s}^{2}}{e^{2}} \sin ^{2} \theta_{W} M_{2}$ at our energy scale.

We have chosen the input values of the parameters in such a way that they reproduce the the SPA1 pole mass values [6] as close as possible. Since the input values of the SPA1 parameters [6] are defined by the $\overline{D R}$ scheme, it is not possible to reproduce exactly the same values as those proposed by SPA1. Note that the lightest neutralino $\left(\tilde{\chi}_{1}^{0}\right)$ is the lightest SUSY-particle (LSP) both in SPA1 and in our parameter choice.

In the numerical calculation we adopt two numerical sets (A) and (B). which are given in Table 1 and 2, respectively. For the fermion masses of the $\mathrm{SM}$ and gauge boson masses, we used the following values (in unit of $\mathrm{GeV}$ ) both for sets (A) and (B),

$$
\begin{aligned}
& M_{W}=80.35, \quad M_{Z}=91.1876, \\
& m_{e}=0.51099906 \times 10^{-3}, \quad m_{\mu}=105.658389 \times 10^{-3}, \quad m_{\tau}=1.7771, \\
& m_{\nu_{e}}=m_{\nu_{\mu}}=m_{\nu_{\tau}}=0, \\
& m_{u}=58.0 \times 10^{-3}, \quad m_{c}=1.5, \quad m_{t}=178.0 \\
& m_{d}=58.0 \times 10^{-3}, \quad m_{s}=92.0 \times 10^{-3}, \quad m_{b}=4.7,
\end{aligned}
$$

while for the strong coupling constant, we used

$$
\alpha_{s}=0.12 \text {. }
$$

The (one-loop improved) mass of Higgs particles, charginos and neutralinos in the set $(\mathrm{A})$ and $(\mathrm{B})$ is given in Table 3 and 4 , respectively.

The set (A) is almost the same as the SPA1a' parameter set, in which not only the heavier chargino $\tilde{\chi}_{2}^{+}$but also the lighter chargino $\tilde{\chi}_{1}^{+}$can decay into 


\begin{tabular}{|c|c|c|c|c|c|c|c|c|c|c|}
\hline $\tan \beta$ & $\mu$ & $M_{1}$ & $M_{2}$ & $M_{3}$ & \multicolumn{2}{|c|}{$M_{A^{0}}$} \\
\hline 10.00 & 399.15 & 100.13 & 157.53 & 610 & \multicolumn{2}{|c|}{431} \\
\hline \hline$m_{\tilde{u}_{1}}$ & $m_{\tilde{u}_{2}}$ & $m_{\tilde{d}_{1}}$ & $m_{\tilde{d}_{2}}$ & $m_{\tilde{e}_{1}}$ & $m_{\tilde{e}_{2}}$ & $m_{\tilde{\nu}_{e}}$ & $\cos \theta_{u}$ & $\cos \theta_{d}$ & $\cos \theta_{e}$ \\
\hline 506.48 & 524.14 & 506.07 & 530.14 & 163.22 & 187.37 & 169.64 & $9.4 \times 10^{-5}$ & $8.5 \times 10^{-4}$ & $9.1 \times 10^{-5}$ \\
\hline \hline$m_{\tilde{c}_{1}}$ & $m_{\tilde{c}_{2}}$ & $m_{\tilde{s}_{1}}$ & $m_{\tilde{s}_{2}}$ & $m_{\tilde{\mu}_{1}}$ & $m_{\tilde{\mu}_{2}}$ & $m_{\tilde{\nu}_{\mu}}$ & $\cos \theta_{c}$ & $\cos \theta_{s}$ & $\cos \theta_{\mu}$ \\
\hline 506.47 & 524.16 & 506.07 & 530.14 & 163.19 & 187.38 & 169.64 & 0.033 & $1.6 \times 10^{-5}$ & 0.019 \\
\hline \hline$m_{\tilde{t}_{1}}$ & $m_{\tilde{t}_{2}}$ & $m_{\tilde{b}_{1}}$ & $m_{\tilde{b}_{2}}$ & $m_{\tilde{\tau}_{1}}$ & $m_{\tilde{\tau}_{2}}$ & $m_{\tilde{\nu}_{\tau}}$ & $\cos \theta_{t}$ & $\cos \theta_{b}$ & $\cos \theta_{\tau}$ \\
\hline 345.37 & 556.78 & 469.43 & 507.15 & 150.07 & 190.39 & 170.02 & 0.5567 & 0.9266 & 0.271 \\
\hline
\end{tabular}

Table 2: The value of the MSSM input parameters for set (B)

\begin{tabular}{|c|c|c|c|c|c|}
\hline$h^{0}$ & $H^{0}$ & $A^{0}$ & \multicolumn{2}{|c|}{$H^{ \pm}$} & \multicolumn{1}{|l|}{} \\
\hline 107.12 & 425.30 & 424.90 & 432.75 \\
\hline \hline$\tilde{\chi}_{1}^{+}$ & $\tilde{\chi}_{2}^{+}$ & $\tilde{\chi}_{1}^{0}$ & $\tilde{\chi}_{2}^{0}$ & $\tilde{\chi}_{3}^{0}$ & $\tilde{\chi}_{4}^{0}$ \\
\hline 184.2 & 421.2 & 97.75 & 184.62 & 398.30 & 413.39 \\
\hline
\end{tabular}

Table 3: The pole mass of Higgs, charginos and neutralinos in set (A)

\begin{tabular}{|c|c|c|c|c|c|}
\hline$h^{0}$ & $H^{0}$ & $A^{0}$ & \multicolumn{2}{|c|}{$H^{ \pm}$} & \multicolumn{1}{|c|}{} \\
\hline 122.50 & 431.40 & 431.0 & \multicolumn{2}{|c|}{438.73} \\
\hline \hline$\tilde{\chi}_{1}^{+}$ & $\tilde{\chi}_{2}^{+}$ & $\tilde{\chi}_{1}^{0}$ & $\tilde{\chi}_{2}^{0}$ & $\tilde{\chi}_{3}^{0}$ & $\tilde{\chi}_{4}^{0}$ \\
\hline 147.08 & 418.8 & 97.61 & 147.4 & 404.0 & 418.8 \\
\hline
\end{tabular}

Table 4: The pole mass of Higgs, charginos and neutralinos in set (B) 
various two bodies because the lighter chargino is heavier than some sleptons. For the set (B), on the other hand, the mass of the lighter chargino $m_{\tilde{\chi}_{1}^{+}}$is smaller than the mass of all sfermions $m_{\tilde{f}}$ as well as the sum $m_{W}+m_{\tilde{\chi}_{1}^{0}}$. This means that the lighter chargino $\tilde{\chi}_{1}^{+}$cannot decay into any two bodies and $\tilde{\chi}_{1}^{+}$has only three body decay modes, $f \bar{f} \tilde{\chi}_{1}^{0}$.

\section{Numerical results}

The one-loop electroweak corrections on various two-body decay widths of the lighter $\tilde{\chi}_{1}^{+}$and heavier chargino $\tilde{\chi}_{2}^{+}$for the parameter set $(\mathrm{A})$ are shown in Table 5 and 6, respectively, where we do not display the decay modes with small branching fraction $\mathrm{Br}<0.1 \%$. While all decays in Table 5 are the electroweak processes, the process $\tilde{\chi}_{2}^{+} \rightarrow \bar{b}_{1}$ in Table 6 gets both the electroweak and QCD corrections through the loop contributions and the photon/gluon emissions. We define $\Gamma \equiv \Gamma_{0}+\delta \Gamma$, where $\Gamma_{0}$ and $\delta \Gamma$ are the improved Born decay width and the one-loop correction, respectively. Note that the improved Born decay width is different from the Born width. We obtain $\Gamma_{0}$ by replacing the tree-level masses by the one-loop renormalized pole-masses presented in Tables 3 and 4 in the tree amplitudes.

\begin{tabular}{lllll}
\hline & $\Gamma_{0}(\mathrm{GeV})$ & $\Gamma(\mathrm{GeV})$ & $\delta \Gamma / \Gamma_{0}$ & $\mathrm{Br}$ \\
\hline$\tilde{\chi}_{1}^{+} \rightarrow \nu_{\tau} \tilde{\tau}_{1}^{+}$ & $3.91 \times 10^{-2}$ & $3.78 \times 10^{-2}$ & $-3.3 \%$ & $50.11 \%$ \\
$\tilde{\chi}_{1}^{+} \rightarrow \nu_{\mu} \tilde{\mu}_{1}^{+}$ & $1.33 \times 10^{-4}$ & $1.19 \times 10^{-4}$ & $-10.2 \%$ & $0.16 \%$ \\
$\tilde{\chi}_{1}^{+} \rightarrow \tau^{+} \tilde{\nu}_{\tau}$ & $1.47 \times 10^{-2}$ & $1.48 \times 10^{-2}$ & $+0.1 \%$ & $19.58 \%$ \\
$\tilde{\chi}_{1}^{+} \rightarrow \mu^{+} \tilde{\nu}_{\mu}$ & $1.06 \times 10^{-2}$ & $1.07 \times 10^{-2}$ & $+1.0 \%$ & $14.24 \%$ \\
$\tilde{\chi}_{1}^{+} \rightarrow e^{+} \tilde{\nu}_{e}$ & $1.06 \times 10^{-2}$ & $1.07 \times 10^{-2}$ & $+1.0 \%$ & $14.22 \%$ \\
$\tilde{\chi}_{1}^{+} \rightarrow W^{+} \tilde{\chi}_{1}^{0}$ & $9.65 \times 10^{-4}$ & $1.28 \times 10^{-3}$ & $+32.3 \%$ & $1.69 \%$ \\
\hline
\end{tabular}

Table 5: One-loop corrections on $\tilde{\chi}_{1}^{+}$decay widths for set $(\mathrm{A})$

In Table 7, we show the one-loop electroweak and QCD corrections to the various 3 -body decay widths of $\tilde{\chi}_{1}^{+}$in the parameter set $(B)$, for which the two-body decays of $\tilde{\chi}_{1}^{+}$are kinematically forbidden. The three-body decay widths of $\tilde{\chi}_{2}^{+}$are not shown, since, decaying dominantly into two bodies, it has extremely small three-body decay branching ratios. Note that for the 


\begin{tabular}{|c|c|c|c|c|}
\hline & $\Gamma_{0}(\mathrm{GeV})$ & $\Gamma(\mathrm{GeV})$ & $\delta \Gamma / \Gamma_{0}$ & $\mathrm{Br}$ \\
\hline$\tilde{\chi}_{2}^{+} \rightarrow \nu_{\tau} \tilde{\tau}_{2}^{+}$ & $1.54 \times 10^{-1}$ & $1.48 \times 10^{-1}$ & $-3.9 \%$ & $4.20 \%$ \\
\hline$\tilde{\chi}_{2}^{+} \rightarrow \nu_{\mu} \tilde{\mu}_{2}^{+}$ & $1.36 \times 10^{-1}$ & $1.46 \times 10^{-1}$ & $+7.5 \%$ & $4.13 \%$ \\
\hline$\tilde{\chi}_{2}^{+} \rightarrow \nu_{e} \tilde{e}_{2}^{+}$ & $1.36 \times 10^{-1}$ & $1.46 \times 10^{-1}$ & $+7.6 \%$ & $4.14 \%$ \\
\hline$\tilde{\chi}_{2}^{+} \rightarrow \tau^{+} \tilde{\nu}_{\tau}$ & $6.89 \times 10^{-2}$ & $5.70 \times 10^{-2}$ & $-17.3 \%$ & $1.61 \%$ \\
\hline$\tilde{\chi}_{2}^{+} \rightarrow \mu^{+} \tilde{\nu}_{\mu}$ & $4.33 \times 10^{-2}$ & $5.38 \times 10^{-2}$ & $+24.2 \%$ & $1.52 \%$ \\
\hline$\tilde{\chi}_{2}^{+} \rightarrow e^{+} \tilde{\nu}_{e}$ & $4.32 \times 10^{-2}$ & $5.37 \times 10^{-2}$ & $+24.4 \%$ & $1.52 \%$ \\
\hline$\tilde{\chi}_{2}^{+} \rightarrow W^{+} \tilde{\chi}_{1}^{0}$ & $1.93 \times 10^{-1}$ & $2.07 \times 10^{-1}$ & $+7.0 \%$ & $5.85 \%$ \\
\hline$\tilde{\chi}_{2}^{+} \rightarrow W^{+} \tilde{\chi}_{2}^{0}$ & $8.66 \times 10^{-1}$ & $9.93 \times 10^{-1}$ & $+14.6 \%$ & $28.12 \%$ \\
\hline$\tilde{\chi}_{2}^{+} \rightarrow Z \tilde{\chi}_{1}^{+}$ & $7.53 \times 10^{-1}$ & $8.56 \times 10^{-1}$ & $+13.7 \%$ & $24.26 \%$ \\
\hline$\tilde{\chi}_{2}^{+} \rightarrow h^{0} \tilde{\chi}_{1}^{+}$ & $5.97 \times 10^{-1}$ & $6.07 \times 10^{-1}$ & $+1.75 \%$ & $17.20 \%$ \\
\hline$\tilde{\chi}_{2}^{+} \rightarrow \bar{b} \tilde{t}_{1}$ & $2.82 \times 10^{-1}$ & $2.57 \times 10^{-1}$ & $\left\{\begin{array}{l}-8.9 \%(\text { ELWK }) \\
+1.8 \%(\mathrm{QCD})\end{array}\right.$ & $7.43 \%$ \\
\hline
\end{tabular}

Table 6: One-loop corrections on $\tilde{\chi}_{2}^{+}$decay widths for set (A)

decay modes involving quarks $\tilde{\chi}_{1}^{+} \rightarrow q \bar{q} \tilde{\chi}_{1}^{0}$, the electroweak and the QCD corrections are separately given.

We should note that the lighter chargino $\tilde{\chi}_{1}^{+}$is the next lightest SUSYparticle (NLSP) in the set (B). Since NLSP must be first produced by accelerators, it is important to study the production processes and the experimental signals of the chargino $\tilde{\chi}_{1}^{+}$. We calculate the full-one-loop electroweak correction for the chargino pair production $e^{+} e^{-} \rightarrow \tilde{\chi}_{1}^{+} \tilde{\chi}_{1}^{-}$at the future linear colliders ( See also [26, 27, 28]). In Fig.1 the energy dependence of the total cross section is shown for the lighter chargino pair production at $e^{+} e^{-}$colliders for the set (B) (and set (A)). The one-loop electroweak correction is of order of $-10 \%$. We note that the radiative correction of this order can be detectable at the proposed linear collider.

By combining the production cross section (Fig.1) and the decay branching ratios (Table 7) for the set (B), we obtain the one-loop corrected cross sections for the direct experimental signals. Fig.2 shows the energy dependence of the cross section for the two types of the chargino signals, $e^{+} e^{-}+$ missing energies and 4-jets + missing energies, at $e^{+} e^{-}$colliders for the set (B). 


\begin{tabular}{cllll}
\hline & $\Gamma_{0}(\mathrm{GeV})$ & $\Gamma(\mathrm{GeV})$ & $\delta \Gamma / \Gamma_{0}$ & $\mathrm{Br}$ \\
\hline$\tilde{\chi}_{1}^{+} \rightarrow e^{+} \nu_{e} \tilde{\chi}_{1}^{0}$ & $4.42 \times 10^{-6}$ & $4.84 \times 10^{-6}$ & $+9.4 \%$ & $20.18 \%$ \\
$\tilde{\chi}_{1}^{+} \rightarrow \mu^{+} \nu_{\mu} \tilde{\chi}_{1}^{0}$ & $4.42 \times 10^{-6}$ & $4.84 \times 10^{-6}$ & $+9.4 \%$ & $20.18 \%$ \\
$\tilde{\chi}_{1}^{+} \rightarrow \tau^{+} \nu_{\tau} \tilde{\chi}_{1}^{0}$ & $6.46 \times 10^{-6}$ & $7.22 \times 10^{-6}$ & $+11.8 \%$ & $30.09 \%$ \\
$\tilde{\chi}_{1}^{+} \rightarrow u \bar{d} \tilde{\chi}_{1}^{0}$ & $3.35 \times 10^{-6}$ & $3.55 \times 10^{-6}$ & $\left\{\begin{array}{l}-0.2 \%(\mathrm{ELWK}) \\
+6.3 \%(\mathrm{QCD})\end{array}\right.$ & $14.81 \%$ \\
$\tilde{\chi}_{1}^{+} \rightarrow c \bar{s} \tilde{\chi}_{1}^{0}$ & $3.33 \times 10^{-6}$ & $3.54 \times 10^{-6}$ & $\left\{\begin{array}{l}-0.2 \%(\mathrm{ELWK}) \\
+6.3 \%(\mathrm{QCD})\end{array}\right.$ & $14.74 \%$ \\
\hline
\end{tabular}

Table 7: One-loop corrections on $\tilde{\chi}_{1}^{+}$decay widths for set (B)

\section{Comments}

The total cross section is a sum of the one-loop corrected cross section and the cross section of the hard photon radiation. The former is negative and large, while the latter is positive and large, originating mainly from the initial radiation. In our calculation for the hard photon radiation, we do not set an energy cut in the upper limit nor any angle cut.

In the calculation of the one-loop correction of the decay widths the situation is almost the same except that in the evaluation of the decay widths the contribution of the real photon (gluon) emission from both initial and final states is important. The correction proportional to the fermion mass $m_{f}$ is expected to be large for the third generation $\tau, t$ and $b$, and an additional enhancement can emerge in the case of large $\tan \beta$ for $\tau$ and $b$. Note that we set $\tan \beta=10$ for both sets (A) and (B). We see these effects in the numerical results presented in Tables 5, 6 and 7, where the branching ratios of the $\tau$ modes are larger than $e$ and $\mu$ modes.

The radiative correction on the chargino pair production in the SPA1a' scenario (set (A)) has been studied by the Wien group [26. Unfortunately, as they adopt different treatment of the photon emission correction, the direct comparison of our result for the full electroweak correction with theirs is not possible. For the comparison we extract the "weak" correction $\Delta \sigma_{\text {weak }}$ defined by

$$
\Delta \sigma_{\text {weak }} \equiv \sigma_{\text {elwk }}-\sigma_{\mathrm{BORN}} * \delta_{\mathrm{QED}}-\sigma_{\text {hard }}^{\text {initial }} .
$$

We find that the result of GRACE/SUSY-loop is consistent with the previous 


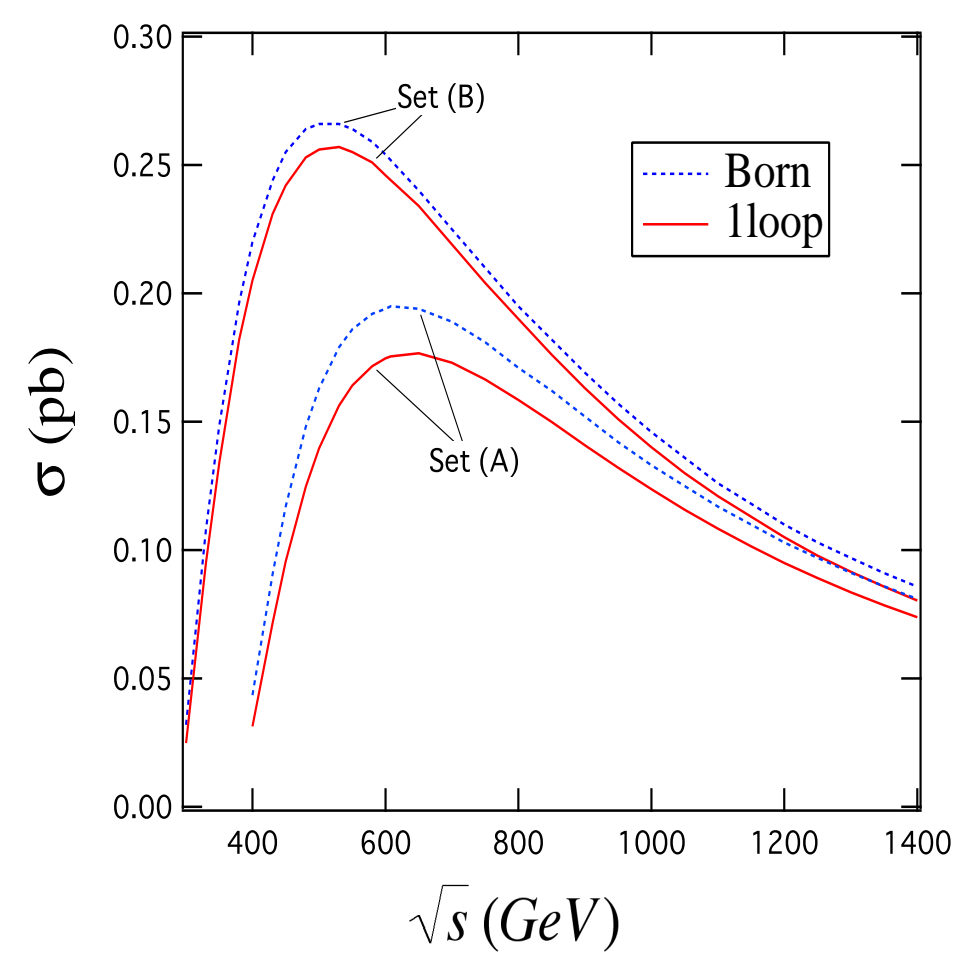

Figure 1: Total cross section for chargino pair production $e^{+} e^{-} \rightarrow \tilde{\chi}_{1}^{+} \tilde{\chi}_{1}^{-}$for the set (A) and set (B). Solid line and dotted line denotes $\sigma_{\text {lloop }}$ and $\sigma_{\mathrm{BORN}}$, respectively.

result [26].

In the parameter setting (A), the produced chargino dominantly decays into two bodies, because the mass of the chargino $m_{\tilde{\chi}_{1}^{+}}(=184.2 \mathrm{GeV})$ is larger than the mass of the lighter charged sleptons, $m_{\tilde{\ell}_{1}}$, and $m_{\tilde{\nu}_{\ell}}$ as well as $m_{W}+m_{\tilde{\chi}_{1}^{0}}$ (see Tables 1 and 3). Since the chargino cannot decay into any squark in two-body decay modes and $\operatorname{BR}\left(\tilde{\chi}_{1}^{+} \rightarrow W^{+} \tilde{\chi}_{1}^{0} \rightarrow q q^{\prime} \tilde{\chi}_{1}^{0}\right)$ is negligibly small (see Table 5), we cannot expect the signals with the quark-jets from the $\tilde{\chi}_{1}^{+}$pair production. We find also that $\operatorname{BR}\left(\tilde{\ell}_{1} \rightarrow \ell \tilde{\chi}_{1}^{0}\right)=1$ and $\operatorname{BR}\left(\tilde{\nu}_{\ell} \rightarrow\right.$ $\left.\nu_{\ell} \tilde{\chi}_{1}^{0}\right)=1$ in the parameter setting (A). This means that the most plausible experimental signal of the chargino pair production is the lepton pair plus the large missing energies. The precise measurement of the energy and the momentum of the $\tau$ leptons is particularly important because $\tau^{+} \tau^{-}$signal is the dominant mode in this case. 

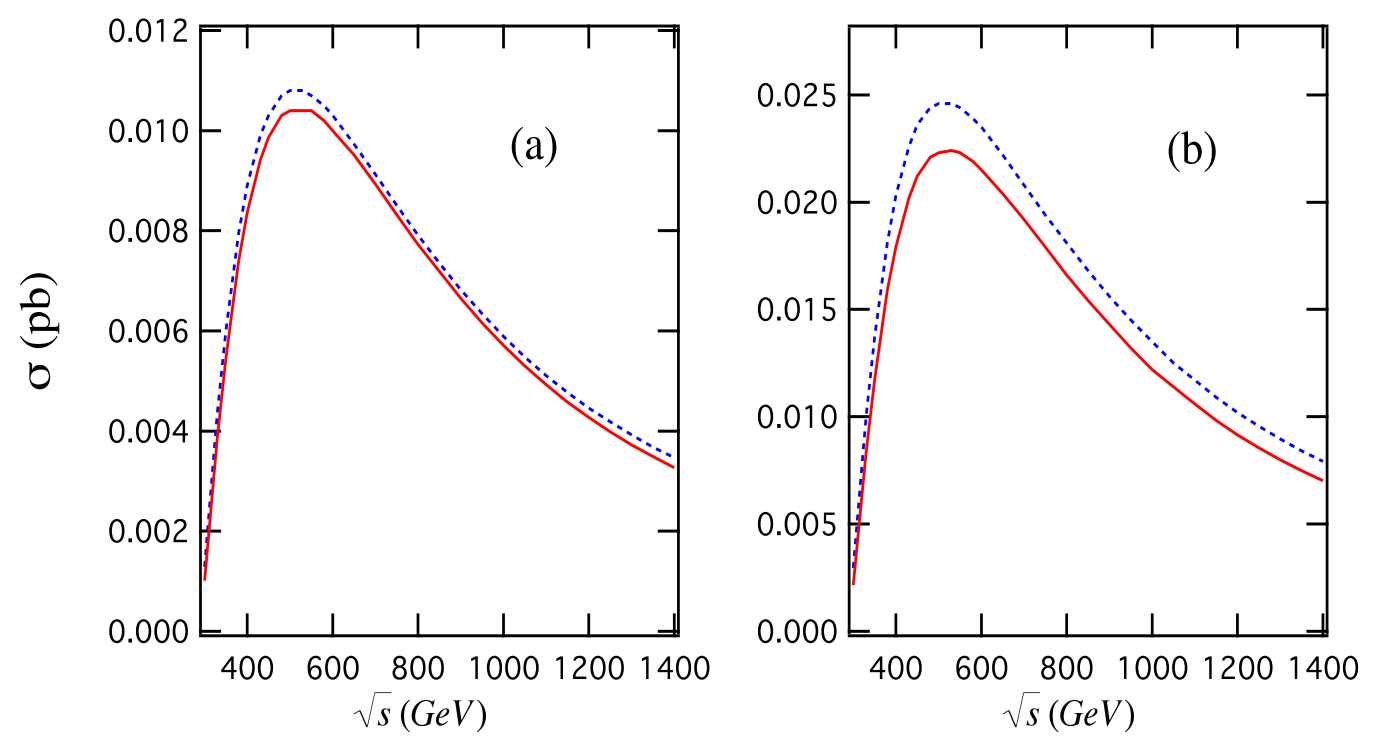

Figure 2: Total cross section for $e^{+} e^{-} \rightarrow \tilde{\chi}_{1}^{+} \tilde{\chi}_{1}^{-} \rightarrow\left(e^{+} \nu_{e} \tilde{\chi}_{1}^{0}\right)\left(e^{-} \bar{\nu}_{e} \tilde{\chi}_{1}^{0}\right)$ (a) and $e^{+} e^{-} \rightarrow \tilde{\chi}_{1}^{+} \tilde{\chi}_{1}^{-} \rightarrow\left(q \bar{q}^{\prime} \tilde{\chi}_{1}^{0}\right)\left(q \bar{q}^{\prime} \tilde{\chi}_{1}^{0}\right)(\mathrm{b})$. Solid line and dotted line denotes $\sigma_{1 \text { loop }}$ and $\sigma_{\mathrm{BORN}}$, respectively.

In the parameter setting $(\mathrm{B})$, the chargino $\tilde{\chi}_{1}^{+}$with $m_{\tilde{\chi}_{1}^{+}}=147.0 \mathrm{GeV}$ is lighter than in set $(\mathrm{A})$ in which $m_{\tilde{\chi}_{1}^{+}}=184.2 \mathrm{GeV}$. We find the apparent shift of the production peak to the lower energy due to the different chargino mass value used in the set $(\mathrm{A})$ and $(\mathrm{B})$. As has been discussed in the previous section, two-body decays of $\tilde{\chi}_{1}^{+}$are kinematically forbidden in the set $(\mathrm{B})$. We can use both types of signals, the lepton pair plus large missing energies and the quark-jets plus large missing energies, for the chargino detection.

We have developed a tool for the full automatic one-loop calculation of the MSSM processes, GRACE/SUSY-loop, which is characterized by the gauge symmetric and the on-shell renormalization scheme, and includes the various self-consistency check schemes. It is certainly a useful tool for the present and future precise analyses of the experimental data.

\section{Acknowledgement}

We would like to thank Y. Shimizu, T. Kaneko, G. Bélanger and F. Boudjema for fruitful discussions. This work was partly supported by Japan Society 
for the Promotion of Science under Grant-in-Aid for Scientific Research (B) (No.17340085). 


\section{Appendix A. Expressions of conterterms}

In this Appendix, we list the expression of the counterterms and the renormalization constants in terms of two-point functions.

The wavefunction renormalization constants are expanded in the one-loop order as

$$
\begin{aligned}
& Z_{X}^{1 / 2}=1+\frac{1}{2} \delta Z_{X}, \\
& Z_{X Y}^{1 / 2}= \begin{cases}1+\frac{1}{2} \delta Z_{X Y}, & X=Y \\
\frac{1}{2} \delta Z_{X Y}, & X \neq Y\end{cases}
\end{aligned}
$$

We use the following abbreviations;

$$
\begin{aligned}
& c_{W}=\cos \theta_{W}, c_{L}=\cos \phi_{L}, c_{R}=\cos \phi_{R}, c_{\alpha}=\cos \alpha, c_{\beta}=\cos \beta \\
& s_{W}=\sin \theta_{W}, s_{L}=\sin \phi_{L}, s_{R}=\sin \phi_{R}, s_{\alpha}=\sin \alpha, s_{\beta}=\sin \beta,
\end{aligned}
$$

where $\phi_{L}$ and $\phi_{R}$ are the mixing angles which diagonalize the chargino mass matrix (see (2.10) of [11]), and $\alpha$ is the mixing angle in the CP even Higgs sector.

gauge sector

$$
\begin{aligned}
\delta Z_{W} & =\operatorname{Re}\left[\Pi_{A A}^{\prime}(0)-2 \frac{c_{w}}{s_{W}} \frac{\Pi_{A Z}(0)}{M_{Z}^{2}}+\frac{\Pi_{W}\left(M_{W}^{2}\right)-c_{W}^{2} \Pi_{Z Z}\left(M_{Z}^{2}\right)}{s_{W}^{2} M_{Z}^{2}}\right], \\
\delta Z_{B} & =\operatorname{Re}\left[\Pi_{A A}^{\prime}(0)+2 \frac{s_{W}}{c_{W}} \frac{\Pi_{A Z}(0)}{M_{Z}^{2}}-\frac{\Pi_{W}\left(M_{W}^{2}\right)-c_{W}^{2} \Pi_{Z Z}\left(M_{Z}^{2}\right)}{c_{W}^{2} M_{Z}^{2}}\right], \\
\delta Z_{g} & =\operatorname{Re}\left[\Pi_{A A}^{\prime}(0)-\frac{2 c_{W}^{2}+1}{c_{W} s_{W}} \frac{\Pi_{A Z}(0)}{M_{Z}^{2}}+\frac{\Pi_{W}\left(M_{W}^{2}\right)-c_{W}^{2} \Pi_{Z Z}\left(M_{Z}^{2}\right)}{s_{W}^{2} M_{Z}^{2}}\right] .
\end{aligned}
$$

Other counterterms appearing in the gauge sector are expressed in terms of $\delta Z_{W}, \delta Z_{B}$ and $\delta Z_{g}$. For example,

$$
\begin{aligned}
\frac{\delta g}{g} & =\delta Z_{g}-\frac{3}{2} \delta Z_{W} \\
\frac{\delta g^{\prime}}{g^{\prime}} & =-\frac{1}{2} \delta Z_{B} \\
\frac{\delta s_{W}}{s_{W}} & =-c_{W}^{2}\left(\frac{\delta g}{g}-\frac{\delta g^{\prime}}{g^{\prime}}\right), \\
\frac{\delta c_{W}}{c_{W}} & =+s_{W}^{2}\left(\frac{\delta g}{g}-\frac{\delta g^{\prime}}{g^{\prime}}\right) .
\end{aligned}
$$


$\underline{\text { fermion sector }}$

$$
\begin{aligned}
\delta m_{f}= & -\operatorname{Re} \Sigma_{f}^{S}\left(m_{f}\right)-m_{f} \operatorname{Re} \Sigma_{f}^{V}\left(m_{f}\right), \\
\delta Z_{f}^{R}= & \operatorname{Re} \Sigma_{f}^{V}\left(m_{f}\right)+\operatorname{Re} \Sigma_{f}^{A}\left(m_{f}\right) \\
& \quad+2 m_{f}\left[\operatorname{Re} \Sigma_{f}^{S \prime}\left(m_{f}\right)+m_{f} \operatorname{Re} \Sigma_{f}^{V \prime}\left(m_{f}\right)\right], \\
\delta Z_{f}^{L}= & \operatorname{Re} \Sigma_{f}^{V}\left(m_{f}\right)-\operatorname{Re} \Sigma_{f}^{A}\left(m_{f}\right) \\
& \quad+2 m_{f}\left[\operatorname{Re} \Sigma_{f}^{S \prime}\left(m_{f}\right)+m_{f} \operatorname{Re} \Sigma_{f}^{V \prime}\left(m_{f}\right)\right] .
\end{aligned}
$$

where the selfenergy function of Dirac fermion $f$ is decomposed as

$$
\Sigma_{f}(\not q) \equiv \Sigma_{f}^{S}\left(q^{2}\right) \mathbf{1}+\Sigma_{f}^{P}\left(q^{2}\right) \gamma_{5}+\Sigma_{f}^{V}\left(q^{2}\right) \not q+\Sigma_{f}^{A}\left(q^{2}\right) \not q \gamma_{5} .
$$

tadpole terms

$$
\delta \mathcal{T}_{i}=T_{\phi_{i}^{0}}^{\text {loop }}, \quad i=1,2
$$

where $\delta \mathcal{T}_{i}$ is the tadpole counterterms.

Higgs sector

$$
\begin{aligned}
& \delta M_{A}^{2}=-\operatorname{Re} \Sigma_{A^{0} A^{0}}\left(M_{A}^{2}\right)+M_{A}^{2} \operatorname{Re} \Sigma_{A^{0} A^{0}}^{\prime}\left(M_{A}^{2}\right), \\
& \left(\begin{array}{c}
\delta Z_{H_{1}} \\
\delta Z_{H_{2}} \\
\frac{\delta v_{1}}{v_{1}} \\
\frac{\delta v_{2}}{v_{2}}
\end{array}\right)=\left(\begin{array}{ccc}
2 c_{\beta}^{2} & & c_{\beta}^{2} \\
-2 s_{\beta}^{2} & & -s_{\beta}^{2} \\
c_{\beta}^{2} & C(i, j) & +\frac{1}{2} \\
-s_{\beta}^{2} & & -\frac{1}{2}
\end{array}\right)\left(\begin{array}{c}
\frac{1}{c_{\beta} s_{\beta}}\left(\frac{\operatorname{Re} \Sigma_{A^{0} Z}\left(M_{A}^{2}\right)}{M_{Z}}\right) \\
C R e \Sigma_{A^{0} A^{0}}^{\prime}\left(M_{A}^{2}\right) \\
C \delta X \\
C c_{2 \beta} \delta Y
\end{array}\right),
\end{aligned}
$$

where

$$
\begin{aligned}
C & =\frac{2}{\sin 2 \beta(\sin 2 \beta+\sin 2 \alpha)} \\
C(1,2) & =3 s_{\beta}^{2} c_{\beta}^{2}-s_{\alpha}^{2} c_{\beta}^{2}+2 s_{\alpha} c_{\alpha} s_{\beta} c_{\beta} \\
C(1,3) & =c_{\beta}^{2}\left(c_{\alpha}^{2}-s_{\beta}^{2}\right) \\
C(2,2) & =3 s_{\beta}^{2} c_{\beta}^{2}-c_{\alpha}^{2} s_{\beta}^{2}+2 s_{\alpha} c_{\alpha} s_{\beta} c_{\beta} \\
C(2,3) & =-s_{\beta}^{2}\left(c_{\alpha}^{2}-s_{\beta}^{2}\right) \\
C(3,2) & =+\frac{1}{2}\left[c_{\beta}^{2}\left(2 s_{\beta}^{2}-1\right)+c_{\alpha}^{2}+2 s_{\alpha} c_{\alpha} s_{\beta} c_{\beta}\right]
\end{aligned}
$$




$$
\begin{aligned}
& C(3,3)=-\frac{1}{2}\left[c_{\beta}^{2}\left(2 s_{\beta}^{2}-1\right)+s_{\alpha}^{2}+2 s_{\alpha} c_{\alpha} s_{\beta} c_{\beta}\right], \\
& C(4,2)=+\frac{1}{2}\left[s_{\beta}^{2}\left(2 c_{\beta}^{2}-1\right)+s_{\alpha}^{2}+2 s_{\alpha} c_{\alpha} s_{\beta} c_{\beta}\right] . \\
& C(4,3)=-\frac{1}{2}\left[s_{\beta}^{2}\left(2 c_{\beta}^{2}-1\right)+c_{\alpha}^{2}+2 s_{\alpha} c_{\alpha} s_{\beta} c_{\beta}\right] .
\end{aligned}
$$

and

$$
\begin{aligned}
\delta X= & \operatorname{Re}\left[\Pi_{A A}^{\prime}(0)+2 c_{W} s_{W} \frac{\Pi_{A Z}(0)}{M_{W}^{2}}\right. \\
+ & \left.\frac{\left(c_{W}^{2}-s_{W}^{2}\right) \Pi_{W}\left(M_{W}^{2}\right)-c_{W}^{4} \Pi_{Z Z}\left(M_{Z}^{2}\right)}{s_{W}^{2} M_{W}^{2}}\right] \\
\delta Y=-\frac{1}{M_{H^{0}}^{2}} & {\left[R e \Sigma_{H^{0} H^{0}}\left(M_{H^{0}}^{2}\right)+\sin ^{2}(\alpha-\beta) \delta M_{A}^{2}+\cos ^{2}(\alpha+\beta) M_{Z}^{2} \delta Z_{Z}\right.} \\
& +\frac{g}{2 M_{W}} \cos (\alpha-\beta)\left[1+\sin ^{2}(\alpha-\beta)\right] \delta T_{H^{0}} \\
& \left.+\frac{g}{2 M_{W}} \sin (\alpha-\beta) \cos ^{2}(\alpha-\beta) \delta T_{h^{0}}\right]
\end{aligned}
$$

with

$$
\delta Z_{Z}=2 c_{W}^{2} \delta Z_{g}-3 c_{W}^{2} \delta Z_{W}-s_{W}^{2} \delta Z_{B} .
$$

The tadpole counterterms for the physical Higgs bosons are defined by

$$
\left(\begin{array}{l}
\delta T_{H^{0}} \\
\delta T_{h^{0}}
\end{array}\right)=\left(\begin{array}{cc}
\cos \alpha & \sin \alpha \\
-\sin \alpha & \cos \alpha
\end{array}\right)\left(\begin{array}{l}
\delta \mathcal{T}_{1} \\
\delta \mathcal{T}_{2}
\end{array}\right)
$$

The counterterm of $\tan \beta$ is then determined by

$$
\delta \tan \beta=-\frac{1}{2} \tan \beta\left(\delta Z_{H_{1}}-\delta Z_{H_{2}}-2 \frac{\delta v_{1}}{v_{1}}+2 \frac{\delta v_{2}}{v_{2}}\right),
$$

while the gauge-boson mass counterterms are given by

$$
\begin{gathered}
\delta M_{W}^{2}=M_{W}^{2}\left[2 \delta Z_{g}-3 \delta Z_{W}+\cos ^{2} \beta \delta Z_{H_{1}}+\sin ^{2} \beta \delta Z_{H_{2}}\right. \\
\left.-2 \cos ^{2} \beta \frac{\delta v_{1}}{v_{1}}-2 \sin ^{2} \beta \frac{\delta v_{2}}{v_{2}}\right], \\
\delta M_{Z}^{2}=M_{Z}^{2}\left[\delta Z_{Z}+\cos ^{2} \beta \delta Z_{H_{1}}+\sin ^{2} \beta \delta Z_{H_{2}}\right. \\
\left.-2 \cos ^{2} \beta \frac{\delta v_{1}}{v_{1}}-2 \sin ^{2} \beta \frac{\delta v_{2}}{v_{2}}\right],
\end{gathered}
$$


$\underline{\text { Chargino sector }}$

$$
\begin{aligned}
\left(\begin{array}{l}
\delta Z_{\lambda^{w}} \\
\delta Z_{\tilde{H}_{1}} \\
\delta Z_{\tilde{H}_{2}}
\end{array}\right) & =\left(\begin{array}{ccc}
1 & \left(s_{L}^{2} c_{R}^{2}+c_{L}^{2} s_{R}^{2}\right) / X & -2 s_{L}^{2} s_{R}^{2} / X \\
1 & \left(2 s_{L}^{2} c_{R}^{2}-c_{L}^{2}-c_{R}^{2}\right) / X & 2 s_{L}^{2} c_{R}^{2} / X \\
1 & \left(2 c_{L}^{2} s_{R}^{2}-c_{L}^{2}-c_{R}^{2}\right) / X & 2 c_{L}^{2} s_{R}^{2} / X
\end{array}\right)\left(\begin{array}{c}
A \\
R e \Sigma_{11}^{A}\left(m_{\tilde{\chi}_{1}^{+}}\right) \\
\operatorname{Re} \Sigma_{22}^{A}\left(m_{\tilde{\chi}_{2}^{+}}\right)
\end{array}\right), \\
\delta M_{2} & =\frac{s_{L} s_{R}\left(\Delta_{22}-\epsilon_{L} \Pi_{22}\right)-c_{L} c_{R}\left(\Delta_{11}-\Pi_{11}\right)}{c_{L}^{2}-s_{R}^{2}}, \\
\delta \mu & =\frac{s_{L} s_{R}\left(\Delta_{11}-\Pi_{11}\right)-c_{L} c_{R}\left(\Delta_{22}-\epsilon_{L} \Pi_{22}\right)}{c_{L}^{2}-s_{R}^{2}},
\end{aligned}
$$

where

$$
\begin{aligned}
X= & s_{L}^{2}-s_{R}^{2} \\
A= & 2 m_{\tilde{\chi}_{1}^{+}}\left[R e \Sigma_{11}^{S \prime}\left(m_{\tilde{\chi}_{1}}\right)+m_{\tilde{\chi}_{1}^{+}} R e \Sigma_{11}^{V \prime}\left(m_{\tilde{\chi}_{1}^{+}}\right)\right]+R e \Sigma_{11}^{V}\left(m_{\tilde{\chi}_{1}^{+}}\right) \\
\Delta_{11}= & +\left(c_{L} c_{R} M_{2}+c_{L} s_{R} M_{W} \frac{\cos \beta}{\sqrt{2}}+s_{L} c_{R} M_{W} \frac{\sin \beta}{\sqrt{2}}\right) \delta Z_{\lambda^{w}} \\
& +\left(c_{L} s_{R} \sqrt{2} M_{W} \cos \beta+s_{L} s_{R} \mu\right) \frac{1}{2} \delta Z_{\tilde{H}_{1}} \\
& +\left(s_{L} c_{R} \sqrt{2} M_{W} \sin \beta+s_{L} s_{R} \mu\right) \frac{1}{2} \delta Z_{\tilde{H}_{2}} \\
& +c_{L} s_{R} \sqrt{2} M_{W} \cos \beta\left(\frac{1}{2} \delta Z_{H_{1}}+\frac{\delta g}{g}-\frac{\delta v_{1}}{v_{1}}\right) \\
& +s_{L} c_{R} \sqrt{2} M_{W} \sin \beta\left(\frac{1}{2} \delta Z_{H_{2}}+\frac{\delta g}{g}-\frac{\delta v_{2}}{v_{2}}\right) \\
\Delta_{22}= & +\left(s_{L} s_{R} M_{2}-s_{L} c_{R} M_{W} \frac{\cos \beta}{\sqrt{2}}-c_{L} s_{R} M_{W} \frac{\sin \beta}{\sqrt{2}}\right) \delta Z_{\lambda^{w}} \\
& +\left(-s_{L} c_{R} \sqrt{2} M_{W} \cos \beta+c_{L} c_{R} \mu\right) \frac{1}{2} \delta Z_{\tilde{H}_{1}} \\
& +\left(-c_{L} s_{R} \sqrt{2} M_{W} \sin \beta+c_{L} c_{R} \mu\right) \frac{1}{2} \delta Z_{\tilde{H}_{2}} \\
& -s_{L} c_{R} \sqrt{2} M_{W} \cos \beta\left(\frac{1}{2} \delta Z_{H_{1}}+\frac{\delta g}{g}-\frac{\delta v_{1}}{v_{1}}\right) \\
& -c_{L} s_{R} \sqrt{2} M_{W} \sin \beta\left(\frac{1}{2} \delta Z_{H_{2}}+\frac{\delta g}{g}-\frac{\delta v_{2}}{v_{2}}\right) \\
& 2 m_{\tilde{\chi}_{1}^{+}}^{2}\left[R e \Sigma_{11}^{S \prime}\left(m_{\tilde{\chi}_{1}^{+}}\right)+m_{\tilde{\chi}_{1}^{+}} R e \Sigma_{11}^{V \prime}\left(m_{\tilde{\chi}_{1}^{+}}\right)\right]-R e \Sigma_{11}^{S}\left(m_{\tilde{\chi}_{1}^{+}}\right),\left(\mathrm{s}_{11}\right. \\
= & (A)
\end{aligned}
$$




$$
\begin{aligned}
\Pi_{22}= & \frac{m_{\tilde{\chi}_{2}^{+}}}{2}\left[\left(s_{L}^{2}+s_{R}^{2}\right) \delta Z_{\lambda^{w}}+c_{R}^{2} \delta Z_{\tilde{H}_{1}}+c_{L}^{2} \delta Z_{\tilde{H}_{2}}\right] \\
& -\operatorname{Re} \Sigma_{22}^{S}\left(m_{\tilde{\chi}_{2}^{+}}\right)-m_{\tilde{\chi}_{2}^{+}} \operatorname{Re} \Sigma_{22}^{V}\left(m_{\tilde{\chi}_{2}^{+}}\right) .
\end{aligned}
$$

$\underline{\text { Neutralino sector }}$

$$
\begin{aligned}
\delta Z_{\lambda} & =\frac{1}{\mathcal{O}_{11}^{2}}\left[\delta Z_{11}-\mathcal{O}_{12}^{2} \delta Z_{\lambda^{w}}-\mathcal{O}_{13}^{2} \delta Z_{\tilde{H}_{1}}-\mathcal{O}_{14}^{2} \delta Z_{\tilde{H}_{2}}\right] \\
\delta M_{1} & =\frac{1}{\mathcal{O}_{11}^{2}}\left[\frac{\delta m_{11}}{\eta_{1}^{* 2}}-\sum_{(p, q) \neq(1,1)} \mathcal{O}_{1 p} \mathcal{O}_{1 q}\left(\delta M_{N}\right)_{p q}-\sum_{(p, q)} \mathcal{O}_{1 p} \mathcal{O}_{1 q} \delta Z_{p}\left(M_{N}\right)_{p q}\right]
\end{aligned}
$$

where $\mathcal{O}$ is the orthogonal matrix which diagonalizes the neutralino mass matrix $M_{N}$

$$
\mathcal{O} M_{N} \mathcal{O}^{t}=\left(\begin{array}{cccc}
m_{\tilde{n}_{1}} & & & \\
& m_{\tilde{n}_{2}} & & \\
& & m_{\tilde{n}_{3}} & \\
& & & m_{\tilde{n}_{4}}
\end{array}\right)
$$

and

$$
\delta M_{N}=\left(\begin{array}{cccc}
\delta M_{1} & 0 & -M_{Z} s_{W} \cos \beta \Delta_{13} & M_{Z} s_{W} \sin \beta \Delta_{14} \\
* & \delta M_{2} & M_{Z} c_{W} \cos \beta \Delta_{23} & -M_{Z} c_{W} \sin \beta \Delta_{24} \\
* & * & 0 & -\delta \mu \\
* & * & * & 0
\end{array}\right)
$$

with

$$
\begin{aligned}
& \Delta_{13}=\frac{\delta M_{Z}^{2}}{2 M_{Z}^{2}}-c_{W}^{2}\left(\frac{\delta g}{g}-\frac{\delta g^{\prime}}{g^{\prime}}\right)-\tan \beta \cos ^{2} \beta \delta \tan \beta \\
& \Delta_{14}=\frac{\delta M_{Z}^{2}}{2 M_{Z}^{2}}-c_{W}^{2}\left(\frac{\delta g}{g}-\frac{\delta g^{\prime}}{g^{\prime}}\right)+\cot \beta \cos ^{2} \beta \delta \tan \beta \\
& \Delta_{23}=\frac{\delta M_{Z}^{2}}{2 M_{Z}^{2}}+s_{W}^{2}\left(\frac{\delta g}{g}-\frac{\delta g^{\prime}}{g^{\prime}}\right)-\tan \beta \cos ^{2} \beta \delta \tan \beta, \\
& \Delta_{24}=\frac{\delta M_{Z}^{2}}{2 M_{Z}^{2}}+s_{W}^{2}\left(\frac{\delta g}{g}-\frac{\delta g^{\prime}}{g^{\prime}}\right)+\cot \beta \cos ^{2} \beta \delta \tan \beta .
\end{aligned}
$$

The phase factor $\eta_{i}$ in (A.36) is to convert the negative mass eigenvalue in (A.37) to positive,

$$
\eta_{i}=\left\{\begin{array}{ll}
1 & m_{\tilde{n}_{i}}>0 \\
i & m_{\tilde{n}_{i}}<0
\end{array} .\right.
$$


$\underline{\text { sfermion sector }}$

For simplicity, we show only the expression for the first generation.

$$
\begin{aligned}
\delta m_{\tilde{f}}^{2} & =-R e \Sigma_{\tilde{f} \tilde{f}}\left(m_{\tilde{f}}^{2}\right), \quad f=u_{1}, u_{2}, d_{1}, d_{2}, e_{1}, e_{2}, \nu_{e}, \\
\delta Z_{\tilde{f} \tilde{f}} & =\Sigma_{\tilde{f} \tilde{f}}^{\prime}\left(m_{\tilde{f}}^{2}\right), \quad f=u_{1}, u_{2}, d_{1}, d_{2}, e_{1}, e_{2}, \nu_{e}, \\
\frac{1}{2} \delta Z_{\tilde{f}_{i} \tilde{f}_{j}} & =-\frac{\Sigma_{\tilde{f}_{i} \tilde{f}_{j}}\left(m_{\tilde{f}_{j}}^{2}\right)}{m_{\tilde{f}_{i}}^{2}-m_{\tilde{f}_{j}}^{2},} \quad i \neq j, \quad f=u, d, e . \\
\delta \theta_{e} & =\frac{\delta m_{\tilde{\nu}_{e}}-\delta\left(M_{W}^{2} \cos 2 \beta-m_{e}^{2}\right)-\cos ^{2} \theta_{e} \delta m_{\tilde{e}_{1}}^{2}-\sin ^{2} \theta_{e} \delta m_{\tilde{e}_{2}}^{2}}{\sin 2 \theta_{e}\left(m_{\tilde{e}_{2}}^{2}-m_{\tilde{e}_{1}}^{2}\right)} \\
\delta \theta_{u} & =\frac{1}{2} \frac{\sum_{\tilde{u}_{1} \tilde{u}_{2}}\left(m_{\tilde{u}_{1}}^{2}\right)+\Sigma_{\tilde{u}_{1} \tilde{u}_{2}}\left(m_{\tilde{u}_{2}}^{2}\right)}{m_{\tilde{u}_{2}}^{2}-m_{\tilde{u}_{1}}^{2}} \\
\delta \theta_{d}=\frac{\text { (A.4.45) }}{\delta\left(\cos ^{2} \theta_{u} m_{\tilde{u}_{1}}^{2}+\sin ^{2} \theta_{u} m_{\tilde{u}_{2}}^{2}-M_{W}^{2} \cos 2 \beta-m_{u}^{2}+m_{d}^{2}\right)-\cos ^{2} \theta_{d} \delta m_{\tilde{d}_{1}}^{2}-\sin ^{2} \theta_{d} \delta m_{\tilde{d}_{2}}^{2}} &
\end{aligned}
$$

$\underline{\text { QCD sector }}$

$$
\begin{aligned}
\delta M_{3} & =-m_{\tilde{g}}\left[\operatorname{Re} \sum_{\tilde{g}}^{S}\left(m_{\tilde{g}}\right)+\operatorname{Re} \Sigma_{\tilde{g}}^{V}\left(m_{\tilde{g}}\right)\right] \\
\delta Z_{\tilde{g}} & =\operatorname{Re} \sum_{\tilde{g}}^{V}\left(m_{\tilde{g}}\right)+2 m_{\tilde{g}}^{2}\left[\operatorname{Re} \sum_{\tilde{g}}^{S \prime}\left(m_{\tilde{g}}\right)+\operatorname{Re} \sum_{\tilde{g}}^{V \prime}\left(m_{\tilde{g}}\right)\right], \\
\delta Z_{\text {gluon }} & =\operatorname{Re} \Pi_{g g}^{\prime}(0), \\
\delta Z_{g_{s}} & =-C\left[\frac{2}{4-d}-\gamma_{E}+\ln (4 \pi)\right] .
\end{aligned}
$$

where $C$ is the finite constant appearing at the one-loop vertex correction as

$$
\left(i V_{\mu \nu \lambda}^{a b c}\right)\left[C\left(\frac{2}{4-d}-\gamma_{E}+\ln (4 \pi)\right)+\cdots\right]
$$




\section{Appendix B. External wavefunction renormal- ization constants}

We list the external wavefunction renormalization constant $\delta Z^{\text {ext }}$ which appears in the amplitude as

$$
\mathcal{M} \sim \frac{1}{2} \delta Z^{e x t} \times(\text { Born amplitude })
$$

$\underline{\text { Gauge bosons }}$

$$
\begin{aligned}
& \delta Z_{W}^{\mathrm{ext}}=\hat{\Pi}_{W}^{\prime}\left(M_{W}^{2}\right)=\Pi_{W}^{\prime}\left(M_{W}^{2}\right)-\delta Z_{W} \\
& \delta Z_{Z}^{\mathrm{ext}}=\hat{\Pi}_{Z Z}^{\prime}\left(M_{Z}^{2}\right)=\Pi_{Z Z}^{\prime}\left(M_{Z}^{2}\right)-\delta Z_{Z Z}
\end{aligned}
$$

Higgs sector

$$
\delta Z_{H^{0}}^{\text {ext }}=\hat{\Sigma}_{H^{0} H^{0}}^{\prime}\left(M_{H^{0}}^{2}\right) .
$$

Since the pole mass of $h^{0}$ and $H^{ \pm}$in one-loop order does not agree with the tree mass, some complication appears.

$$
\delta Z_{h^{0}}^{\text {ext }}=\frac{1}{1-\hat{\Sigma}_{h h}^{* \prime}\left(M_{h^{0}}^{2}, m_{h^{0}}^{2}, m_{H^{0}}^{2}\right)}-1 \approx \hat{\Sigma}_{h h}^{* \prime}\left(M_{h^{0}}^{2}, m_{h^{0}}^{2}, m_{H^{0}}^{2}\right)
$$

where

$$
\begin{aligned}
\hat{\Sigma}_{h h}^{* \prime}\left(M_{h^{0}}^{2}\right) \equiv & \left.\frac{\partial}{\partial q^{2}} \hat{\Sigma}_{h h}\left(q^{2}\right)\right|_{M_{h^{0}}^{2}} \\
& +\left.\frac{\partial}{\partial q^{2}}\left[\frac{\hat{\Sigma}_{H h}^{2}\left(q^{2}\right)}{q^{2}-m_{H^{0}}^{2}-\hat{\Sigma}_{H H}\left(q^{2}\right)}\right]\right|_{M_{h^{0}}^{2}}
\end{aligned}
$$

and $M_{h^{0}}$ is the one-loop improved pole mass of $h^{0}$. The renormalized selfenergy functions are defined by

$$
\begin{aligned}
\hat{\Sigma}\left(q^{2}\right)_{h h} & =\Sigma\left(q^{2}\right)_{h h}+\delta M_{h h}^{2}-q^{2}\left(\sin ^{2} \alpha \operatorname{Re} \delta Z_{H_{1}}+\cos ^{2} \alpha \operatorname{Re} \delta Z_{H_{2}}\right), \\
\hat{\Sigma}\left(q^{2}\right)_{H H} & =\Sigma\left(q^{2}\right)_{H H}+\delta M_{H H}^{2}-q^{2}\left(\cos ^{2} \alpha \operatorname{Re} \delta Z_{H_{1}}+\sin ^{2} \alpha \operatorname{Re} \delta Z_{H_{2}}\right), \\
\hat{\Sigma}\left(q^{2}\right)_{H h} & =\Sigma\left(q^{2}\right)_{H h}+\delta M_{H h}^{2}-q^{2} \cos \alpha \sin \alpha\left(\operatorname{Re} \delta Z_{H_{2}}-\operatorname{Re} \delta Z_{H_{1}}\right),
\end{aligned}
$$


with

$$
\begin{aligned}
\delta M_{h h}^{2}= & \cos ^{2}(\alpha-\beta) \delta M_{A}^{2} \\
& -\frac{g}{2 M_{W}} \cos (\alpha-\beta) \sin ^{2}(\alpha-\beta) T_{H^{0}}^{\text {lop }} \\
& -\frac{g}{2 M_{W}} \sin (\alpha-\beta)\left[1+\cos ^{2}(\alpha-\beta)\right] T_{h^{0}}^{\text {loop }} \\
& +M_{Z}^{2}\left[\sin ^{2}(\alpha+\beta)\left(\delta Z_{Z}+\delta Z_{H_{1}}+\delta Z_{H_{2}}\right)\right. \\
& \left.\quad+\sin (\alpha+\beta) \sin (\alpha-\beta)\left(\delta Z_{H_{1}}-\delta Z_{H_{2}}\right)\right] \\
& +2 \sin (\alpha+\beta)[\sin \beta \cos \beta \cos (\alpha+\beta)-\sin \alpha \cos \beta] M_{Z}^{2} \frac{\delta v_{1}}{v_{1}} \\
& -2 \sin (\alpha+\beta)[\sin \beta \cos \beta \cos (\alpha+\beta)+\cos \alpha \sin \beta] M_{Z}^{2} \frac{\delta v_{2}}{v_{2}} \\
\delta M_{H H}^{2}= & -R e \Sigma_{H H}\left(M_{H}^{2}\right)+M_{H^{0}}^{2}\left(\cos { }^{2} \alpha \delta Z_{H_{1}}+\sin { }^{2} \alpha \delta Z_{H_{2}}\right), \\
\delta M_{H h}^{2}= & -\sin (\beta-\alpha) \cos (\beta-\alpha) \delta M_{A}^{2} \\
& +\frac{g}{2 M_{W}} \sin (\beta-\alpha) T_{H^{0}}^{\mathrm{loop}}+\frac{g}{2 M_{W}} \cos { }^{3}(\alpha-\beta) T_{h^{0}}^{\mathrm{loop}} \\
& -M_{Z}^{2}\left[\sin (\alpha+\beta) \cos (\alpha+\beta)\left(\delta Z_{Z}+\delta Z_{H_{1}}+\delta Z_{H_{2}}\right)\right. \\
& \left.\quad+\sin \alpha \cos \alpha\left(\delta Z_{H_{1}}-\delta Z_{H_{2}}\right)\right] \\
& +\left[\frac{1}{2} \sin (2 \alpha+2 \beta)(1+\cos 2 \beta)-\frac{\sin 2 \alpha \cos 2 \alpha \sin 2 \beta}{\sin (2 \alpha-2 \beta)}\right] M_{Z}^{2} \frac{\delta v_{1}}{v_{1}} \\
& +\left[\frac{1}{2} \sin (2 \alpha+2 \beta)(1-\cos 2 \beta)+\frac{\sin 2 \alpha \cos 2 \alpha \sin 2 \beta}{\sin (2 \alpha-2 \beta)}\right] M_{Z}^{2} \frac{\delta v_{2}}{v_{2}} .
\end{aligned}
$$

The expressions (B.5) and (B.6) agree with those given in [25].

$$
\delta Z_{H^{ \pm}}^{\mathrm{ext}}=\frac{1}{1-\hat{\Sigma}_{H^{ \pm} H^{ \pm}}^{* \prime}\left(M_{H^{ \pm}}^{2}\right)}-1 \sim \hat{\Sigma}_{H^{ \pm} H^{ \pm}}^{* \prime}\left(M_{H^{ \pm}}^{2}\right)
$$

where

$$
\begin{aligned}
\hat{\Sigma}_{H^{ \pm} H^{ \pm}}^{* \prime}\left(q^{2}\right)= & \left.\frac{\partial}{\partial q^{2}} \hat{\Sigma}_{H \pm H^{ \pm}}\left(q^{2}\right)\right|_{M_{H^{ \pm}}^{2}} \\
& +\left.\frac{\partial}{\partial q^{2}}\left[\frac{\hat{\Sigma}_{H^{ \pm} G^{ \pm}}^{2}\left(q^{2}\right)}{q^{2}-m_{H^{ \pm}}^{2}-\hat{\Sigma}_{H^{ \pm} H^{ \pm}}\left(q^{2}\right)}\right]\right|_{M_{H^{ \pm}}^{2}}
\end{aligned}
$$

The renormalized selfenergy functions appearing in (B.14) are given by

$$
\begin{aligned}
& \hat{\Sigma}\left(q^{2}\right)_{H^{ \pm} H^{ \pm}}=\Sigma\left(q^{2}\right)_{H^{ \pm} H^{ \pm}}+\delta M_{H^{ \pm} H^{ \pm}}^{2}-q^{2}\left(s_{\beta}^{2} \operatorname{Re} \delta Z_{H_{1}}+c_{\beta}^{2} \operatorname{Re} \delta Z_{H_{2}}\right), \\
& \hat{\Sigma}\left(q^{2}\right)_{H^{ \pm} G^{ \pm}}=\Sigma\left(q^{2}\right)_{H^{ \pm} G^{ \pm}}+\delta M_{H^{ \pm} G^{ \pm}}^{2}-q^{2} c_{\beta} s_{\beta}\left(\operatorname{Re} \delta Z_{H_{2}}-\operatorname{Re} \delta Z_{H_{1}}\right),
\end{aligned}
$$


with

$$
\begin{aligned}
& \delta M_{H^{ \pm} H^{ \pm}}^{2}=\delta M_{A^{0} A^{0}}^{2}+M_{W}^{2}\left(\delta Z_{x}-2 c_{\beta}^{2} \frac{\delta v_{1}}{v_{1}}-2 s_{\beta}^{2} \frac{\delta v_{2}}{v_{2}}\right), \\
& \delta M_{H^{ \pm} G^{ \pm}}^{2}=\delta M_{G^{0} A^{0}}^{2}-c_{\beta} s_{\beta} M_{W}^{2}\left(\frac{\delta v_{1}}{v_{1}}-\frac{\delta v_{2}}{v_{2}}\right) .
\end{aligned}
$$

In the one-loop order, (B.5) and (B.14) become

$$
\begin{aligned}
\delta Z_{h h}^{\text {ext }} & =\hat{\Sigma}_{h^{0} h^{0}}^{\prime}\left(M_{h^{0}}^{2}\right), \\
\delta Z_{H^{ \pm}}^{\text {ext }} & =\hat{\Sigma}_{H \pm H^{ \pm}}^{\prime}\left(M_{H^{ \pm}}^{2}\right) .
\end{aligned}
$$

$\underline{\text { Chargino }}$

$$
\delta Z_{\tilde{\chi}_{2}^{+}}^{\text {ext }}=2 M_{\tilde{\chi}_{2}^{+}}\left(\Sigma_{2}^{S \prime}\left(M_{\tilde{\chi}_{2}^{+}}^{2}\right)+M_{\tilde{\chi}_{2}^{+}} \Sigma_{2}^{V \prime}\left(M_{\tilde{\chi}_{2}^{+}}^{2}\right)\right)+\Sigma_{2}^{V}\left(M_{\tilde{\chi}_{2}^{+}}^{2}\right)-\frac{1}{2}\left(\delta Z_{22}^{R}+\delta Z_{22}^{L}\right),
$$

where the chargino selfenergy functions are decomposed as (A.14). In terms of the renormalization constants introduced in section 2, the chargino wavefunction renormalization constants are given by

$$
\begin{aligned}
& \delta Z_{22}^{L}=\sin \phi_{L}^{2} \delta Z_{\lambda^{w}}+\cos \phi_{L}^{2} \delta Z_{\tilde{H}_{2}}, \\
& \delta Z_{22}^{R}=\sin \phi_{R}^{2} \delta Z_{\lambda^{w}}+\cos \phi_{R}^{2} \delta Z_{\tilde{H}_{1}} .
\end{aligned}
$$

$\underline{\text { Neutralino }}$

$$
\delta Z_{\tilde{\chi}_{i}^{0}}^{\text {ext }}=2 M_{\tilde{\chi}_{i}^{0}}\left[\sum_{i i}^{S \prime}\left(M_{\tilde{\chi}_{i}^{0}}^{2}\right)+M_{\tilde{\chi}_{i}^{0}} \sum_{i i}^{V \prime}\left(M_{\tilde{\chi}_{i}^{0}}^{2}\right)\right]+\Sigma_{i i}^{V}\left(M_{\tilde{\chi}_{i}^{0}}^{2}\right)-\delta Z_{i i} .
$$

where $i=2,3,4$ and the selfenergy function of Majorana particles is decomposed as

$$
\Sigma_{f}(\not) \equiv \Sigma_{f}^{S}\left(q^{2}\right) \mathbf{1}+\Sigma_{f}^{V}\left(q^{2}\right) \not 1 .
$$

The neutralino wavefunction renormalization constant appearing in (B.23) is expressed in terms of the basic renormalization constants introduced in section 2 as

$$
\delta Z_{i i}=\sum_{k}(\operatorname{Re} \delta Z)_{k}\left(\mathcal{O}_{N}\right)_{i k}\left(\mathcal{O}_{N}\right)_{i k}, \quad \text { no sum over } i,
$$

where

$$
\delta Z_{k} \equiv\left(\delta Z_{\lambda}, \delta Z_{\lambda^{w}}, \delta Z_{\tilde{H}_{1}}, \delta Z_{\tilde{H}_{2}}\right)
$$


and $\mathcal{O}$ is the orthogonal matrix which diagonalizes the neutralino mass matrix. See (A.37).

Note that for unstable particles, even if the residue condition is imposed on the propagator at the pole position, there is a non-vanishing $\delta Z^{\text {ext }}$ which is ultraviolet-finite and purely imaginary. For example, we can easily check that $\delta Z_{\tilde{\chi}_{1}^{+}}^{\text {ext }}$ which is obtained from (B.21) by changing the index 2 to 1 , is purely imaginary. We can neglect such contributions if perturbation works.

\section{References}

[1] For review see, for example, P. Fayet and S. Ferrara, Phys. Rep. 32 (1977) 249; H. Haber and G. Kane, Phys. Rep. 117 (1985) 75.

[2] H.P. Nilles, Phys. Rep. 110 (1984) 1.

[3] S.L. Glashow, Nucl. Phys. 22 (1961) 579; S. Weinberg, Phys. Rev. Lett. 19 (1967) 1264; A. Salam, Proceedings of the Nobel symposium, 1968, Lerum, Sweden.

[4] J. Fujimoto et al., Comp. Phys. Comm. 111 (1998) 185.

[5] J. Fujimoto et al., Comp. Phys. Comm. 153 (2003) 106.

[6] J.A. Aguilar-Saavedra et al., Eur. Phys. J. C46 (2006) 43.

[7] T. Ishikawa et al., KEK Report 92-19, 1993, The GRACE manual v1.0; F. Yuasa et al., Prog. Theor. Phys. Suppl. 138 (2000) 18, hep-ph/0007053.

[8] J. Fujimoto, T. Ishikawa, M. Jimbo, T. Kaneko, T. Kon and M. Kuroda, Proceedings of the XVIIth International Workshop on High Energy Physics and Quantum Field Theory, (2004) 26, hep-ph/0402144.

[9] J. Fujimoto, T. Ishikawa, M. Jimbo, T. Kon and M. Kuroda, Nucl. Instrum. Methods Phys. Res. A 534 (2004) 246.

[10] J. Fujimoto et al., Nucl. Phys. Proc. Suppl. 157 (2006) 157.

[11] M. Kuroda, KEK-CP 080 (1999), hep-ph/9902340]. 
[12] K. Hikasa, SUSY manuscript version July 5, 1995, (1995), [unpublished].

[13] J. Rosiek, Phys. Rev. D41 (1990) 3464; erratum KA-TA-8-1995.

[14] P.H. Chankowski, S. Pokorski and J. Rosiek, Nucl. Phys. B423 (1994) 437.

[15] Y. Yamada, Phys. Lett B530 (2002) 174.

[16] J. Guasch, J. Sola and W. Hollik, Phys. Lett. B437 (1998) 88.

[17] G. Bélanger et al., Phys. Rep. 430 (2006) 117.

[18] A. Yamada, Phys. Lett. B263 (1991) 233; Z. Phys. C61 (1994) 247.

[19] A. Dabelstein, Z. Phys. C67 (1995) 495.

[20] D. Pierce and A. Papadopoulos, Phys. Rev. D50 (1994) 565; Nucl. Phys. B430 (1994) 278.

[21] S. Alam, K. Hagiwara, S. Kanemura, R. Szalapski and Y. Umeda, Phys. Rev. D62 (2000) 095011.

[22] W. Hollik, E. Kraus and M. Roth, C. Rupp, K. Sibold, D. Stöckinger, Nucl. Phys. B639 (2002) 3.

[23] J. Guasch, W. Hollik, J. Sola, JHEP 0210 (2002) 040, hep-ph/0207364.

[24] T. Fritzsche and W. Hollik, Eur. Phys. J. C24 (2002) 619.

[25] S. Heinemeyer, W. Hollik, J. Rosiek and G. Weiglein, Eur. Phys. J. C19 (2001) 535 .

[26] W. Öller, H. Eberl and W. Majerotto, Phys. Rev. D71 (2005) 115002.

[27] W. Killian, J. Reuter and T. Robens, Eur. Phys. J. C48 (2006) 389.

[28] W. Kilian, J. Reuter and T. Robens, DESY 06-189 (2006), hep-ph/0610425. 